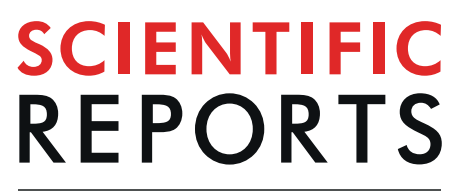

natureresearch

\title{
OPEN Role of mineral nutrition in alleviation of heat stress in cotton plants grown in glasshouse and field conditions
}

Received: 10 January 2019

Accepted: 18 July 2019

Published online: 10 September 2019
Muhammad Sarwar ${ }^{1}$, Muhammad Farrukh Saleem ${ }^{2}$, Najeeb Ullah $\left(\mathbb{1}^{3}{ }^{3}\right.$, Shafaqat Ali $^{4}$, Muhammad Rizwan ${ }^{4}$, Muhammad Rizwan Shahid ${ }^{5}$, Mohammed Nasser Alyemeni ${ }^{6}$, Saud A. Alamri' ${ }^{6}$ Parvaiz Ahmad $\mathbb{1}^{6,7}$

Coincidence of high temperature with terminal reproductive pheno-stages of cotton is chief constraint to achieve yield potential. This high temperature interfere plant defensive system, physiological process, water relations and lint yield production. In this study, we modulated the detrimental outcomes of heat stress on cotton through the foliar spray of nutrients. Cotton crop was exposed to sub-optimal and supra-optimal thermal regimes for a period of one week at squaring, flowering and boll formation stages under glass house and field conditions. Foliar spray of potassium (K$1.5 \%)$, zinc ( $\mathrm{Zn}-0.2 \%)$ and boron (B-0.1\%) were applied at three reproductive stages one day prior to expose high temperature regimes. High temperature increased lipid membrane damage through increased malondialdehyde (MDA) contents in cotton leaves. High temperature stress also reduced leaf chlorophyll contents, net photosynthetic rate, stomatal conductance, water potential, averaged boll weight $(\mathrm{g})$ and seed cotton yield per plant. Various nutrients variably influenced growth and physiology of heat-stressed cotton plants. Zinc outclassed all other nutrients in increasing leaf SOD, CAT, POX, AsA, TPC activity, chlorophyll contents, net photosynthetic rate, stomatal conductance, water potential, boll weight and seed cotton yield per plant. For example, zinc improved seed cotton yield under supra-optimal thermal regime by $17 \%$ and under sub-optimal thermal regime by $12 \%$ of glasshouse study while $19 \%$ under high temperature sowing dates of field study than the water treated plants under the same temperatures. Conclusively, increasing intensities of temperature adversely affected the recorded responses of cotton and exogenous application of $Z n$ efficaciously alleviated heat induced perturbations. Moreover, exogenous nutrients mediated upregulations in physiochemical attributes induced heat tolerance at morphological level.

Temperature is prophesied to rise by $5.8^{\circ} \mathrm{C}$ till 2100 and $2.6^{\circ} \mathrm{C}$ up to 2050 owing to global warming ${ }^{1}$. Heat waves along with more number of warm days and nights have been increased in most part of the world ${ }^{2}$. Cotton crop being native of semiarid regions is highly prone to confront with high temperature at the terminal reproductive stages $^{3}$. Coincidence of high temperature with reproductive stages of cotton is a chief hindrance to accomplish yield potential in sub-continent. Since, temperature rises to $47^{\circ} \mathrm{C}$ in May-June while accompanying high humidity in July-August develops a death-valley for cotton influencing all reproductive stages of cotton crop ${ }^{4}$. Heat stress mediated impairment in biosynthesis of antioxidants escalates the synthesis of reactive oxygen species

\footnotetext{
${ }^{1}$ Agronomic Research Institute, Ayub Agricultural Research Institute, Faisalabad, Pakistan. ${ }^{2}$ Department of Agronomy, University of Agriculture Faisalabad, 38040, Faisalabad, Pakistan. ${ }^{3}$ Queensland Alliance for Agriculture and Food Innovation | Centre for Plant Science, University of Queensland Wilsonton Heights, Toowoomba, QLD 4350, Australia. ${ }^{4}$ Department of Environmental Sciences and Engineering, Government College University Allamalqbal Road, 38000, Faisalabad, Pakistan. ${ }^{5}$ Institute of Soil and Environmental Sciences, University of Agriculture, Faisalabad, 38000, Pakistan. ${ }^{6}$ Department of Botany and Microbiology, College of Science, King Saud University, Riyadh, Saudi Arabia. ${ }^{7}$ Department of Botany, S.P. College, Maulana Azad Road, Srinagar, Jammu and Kashmir, 190001, India. Correspondence and requests for materials should be addressed to S.A. (email: shafaqataligill@ yahoo.com) or P.A. (email: parvaizbot@yahoo.com)
} 


\begin{tabular}{|l|l|l|l|l|l|l|}
\hline \multirow{2}{*}{ Growth phases } & \multicolumn{2}{|l}{2012} & \multicolumn{2}{l|}{2013} & \multicolumn{2}{l|}{} \\
\cline { 2 - 7 } & April & May & June & April & May & June \\
\hline Squaring (initiation) & $35.0-$ & $38.5-$ & $34.0-$ & $33.0-$ & $39.0-44^{\circ} \mathrm{C}$ & $34.0-$ \\
& $38.0^{\circ} \mathrm{C}$ & $45.0^{\circ} \mathrm{C}$ & $39.0^{\circ} \mathrm{C}$ & $39.0^{\circ} \mathrm{C}$ & & $37.0^{\circ}$ \\
\hline Flowering (initiation) & $38.0-$ & $43.5^{\circ}-$ & $33.0-$ & $37.0-$ & $39.0-$ & $31.8^{\circ}$ \\
& $44.0^{\circ} \mathrm{C}$ & $45.0^{\circ} \mathrm{C}$ & $38.0^{\circ} \mathrm{C}$ & $45.7^{\circ} \mathrm{C}$ & $41.3^{\circ} \mathrm{C}$ & $37.0^{\circ} \mathrm{C}$ \\
\hline \multirow{2}{*}{ Boll formation } & $38.0-$ & $42.5^{\circ}-$ & $33.0-$ & $33.9-$ & $32.0-$ & $35.5-$ \\
& $41.0^{\circ} \mathrm{C}$ & $46.0^{\circ} \mathrm{C}$ & $39.0^{\circ} \mathrm{C}$ & $44.9^{\circ} \mathrm{C}$ & $39.2^{\circ} \mathrm{C}$ & $37.2^{\circ} \mathrm{C}$ \\
\hline
\end{tabular}

Table 1. Variable temperatures (thermal regimes) during one week of study under field conditions at three reproductive stages of cotton. Optimal $=34-38^{\circ} \mathrm{C}$, sub optimal $=39-41^{\circ} \mathrm{C}$ and supra-optimal up to $46^{\circ} \mathrm{C}$ Maximum temperature ranges of treatment period - one week. At squaring stage, temperature of the May sown crop was raised to supra optimal condition during both years of study. Flowering stage of April-2012, May-2012 and April-2013 sown crops experienced heat stress period, while sub optimal temperature prevailed at same stage of May-2013 sown crop. Heat stress periods were also observed at boll formation May-2012 and April2013 sown crops while boll formation stage of April-2012 sown crop faced sub optimal conditions. June planted crop faced optimal temperatures at all reproductive stages during both years of study and thus considered optimal or control sowing date.

(ROS) and thus induces oxidative stress ${ }^{4,5}$. Consequently, a cascade of reactions consequence into plethora of ROS that aggravate lipid peroxidation, excessive synthesis of malondialdehyde contents (MDA) ${ }^{6}$ and ultimately disruption in stability of membranes. Henceforth, the biosynthesis of chlorophyll contents decreases while degradation aggravates under heat triggered oxidative stress ${ }^{7}$. Whereas, impairment in biosynthesis of chlorophyll ultimately down regulates photosynthesis, translocation of assimilates to reproductive organs and accelerates senescence ${ }^{8,9}$. Moreover, heat stress also disrupts stomatal movement and ultimately consequence into poor gaseous exchange. Cotton leaves depict optimum stomatal conductance in temperature ranges $28-30.1{ }^{\circ} \mathrm{C}^{10}$ which starts to decline as temperature rises to $36^{\circ} \mathrm{C}$. Contrarily, as the temperature rises above $40^{\circ} \mathrm{C}$, stomata remain open and photosynthesis is impaired even if soil is well watered. Concurrently, high temperature stress mediated incongruities in stomatal movements disrupts the water relations resulting into reduction of growth ${ }^{11,12}$. Perturbations in biochemical attributes ultimately affect morphological attributes. The optimum temperature for development of boll ranges $25.5^{\circ} \mathrm{C}-29.5^{\circ} \mathrm{C}$. While, boll weight is adversely affected as temperature rises above $25.5^{\circ} \mathrm{C}-29.5^{\circ} \mathrm{C}^{13}$. Similarly, each $1^{\circ} \mathrm{C}$ rise of temperature above day maximum temperature decreases seed cotton yield by $110 \mathrm{~kg} \mathrm{ha}^{-114}$. Abiotic stresses along with oxidative stress and nutrient deficiency are the major causes of yield reduction throughout world ${ }^{15}$. Exogenous application of nutrients might prove a potent tool to alleviate deleterious impacts of heat ${ }^{16}$. Moreover, heat triggered decrease in uptake of nutrients from soil under heat stress further enhance the importance of exogenous supply of nutrients. Moreover, foliar applied nutrients produce higher yield and better-quality produce on alkaline calcareous soils ${ }^{17}$. Potassium and zinc are immobile within calcareous soils while B availability is also an issue in these soils ${ }^{18-20}$. Contrarily, potassium, zinc and boron are required in high quantity during the stress conditions. Potassium, zinc and boron modulates biochemical changes through antioxidant enzymes ${ }^{21-23}$ whereas, the exogenous application of potassium, zinc and boron up regulates the biosynthesis of chlorophyll which ultimately delays senescence enhances their quantity; consequently, improves the photosynthetic rate and the photosynthetic enzymes ${ }^{24}$. Likewise, potassium and zinc mediated regulations in water relations confer heat tolerance by sustaining water and osmotic potential of cell under stress conditions. While, boron availability enhances stomatal opening and thus regulate gaseous exchange under stressed environment ${ }^{25,26}$.

Therefore, considering the crucial role of macro $(\mathrm{K})$ and micro nutrients ( $\mathrm{Zn}$ and $\mathrm{B}$ ) in protecting crops from extensive range of abiotic stresses, exogenous application under stress conditions might prove a potent tool to alleviate adverse impact of stress. The present study compares the potential role of foliar spray of macronutrient (potassium) in photosynthesis, regulation of water relations and stomatal conductance and micronutrients (zinc and boron) in reproduction and antioxidants. These physiochemical regulations might induce tolerance in morphological attributes of cotton crop exposed to different thermal regimes at squaring, flowering and boll formation. Hence, series of glasshouse and field experiments were conducted with objectives to (1) see the effect of different temperature regimes on leaf physiology and lint yield of cotton and to (2) reveal the role of macro and micro nutrients $(\mathrm{K}, \mathrm{Zn}$ and $\mathrm{B})$ for alleviation the impact of high temperature stress.

\section{Materials and Methods}

Glasshouse experiment. The glasshouse experiment was conducted at University of Agriculture Faisalabad (UAF), Pakistan. The experiment was performed during summer 2012. The seed of medium heat tolerant variety (AA-802) was collected from Ali Akbar Enterprises for this study. Soil properties and growth condition were same as have been reported in an earlier study ${ }^{4}$. Four seeds were sown at $2 \mathrm{~cm}$ depth had 12 hours pre-soaking in tap water. At four leaf stage of the seedlings, extra plants were thinned left only one plant in each pot. Treatments were comprised of optimal temperature $\left(32 / 20 \pm 2{ }^{\circ} \mathrm{C}\right.$ day/night temperature or no stress), sub-optimal temperature $\left(38 / 24^{\circ} \mathrm{C} \pm 2{ }^{\circ} \mathrm{C}\right.$ or medium intensity heat stress) and supra-optimal temperature $\left(45 / 30^{\circ} \mathrm{C} \pm 2{ }^{\circ} \mathrm{C}\right.$ or high intensity heat stress); exogenously applied nutrients viz. water spray (control), foliar spray of $\mathrm{K} @ 1.5 \%$, foliar spray of $\mathrm{Zn} @ 0.2 \%$ and foliar spray of B @ $0.1 \%$. One day before shifting the pots to medium and high temperature chambers, the plants were sprayed with either of water (control), potassium (1.5\%), zinc ( $0.2 \%)$ and boron $(0.1 \%)$. Foliar concentrations of these nutrients were optimized in the preliminary glasshouse and field experiments (data 
(a)

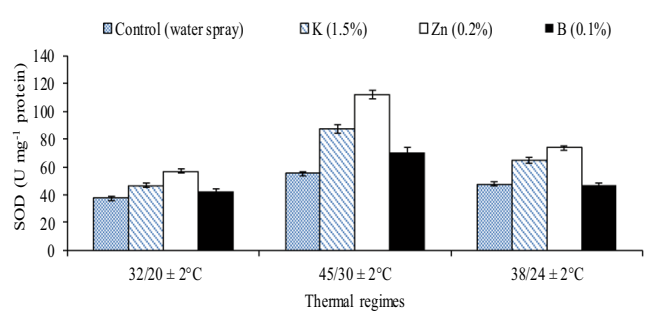

(c)

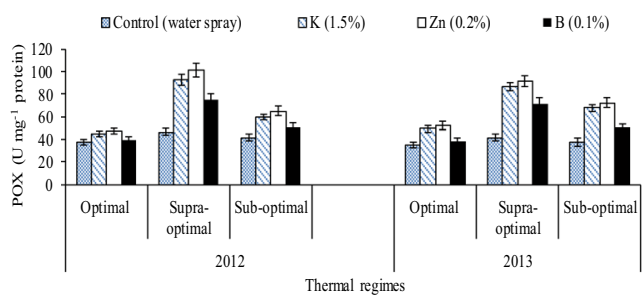

(e)

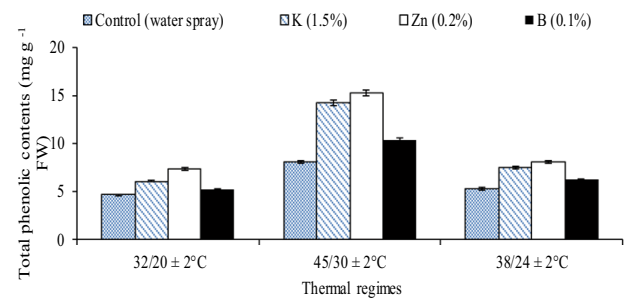

(b)

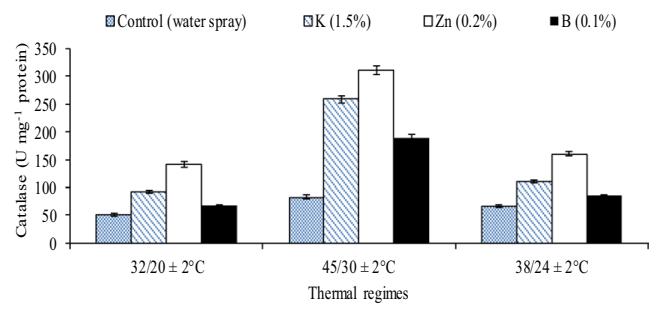

(d)

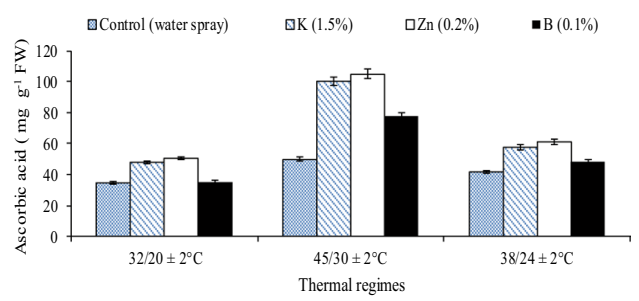

(f)

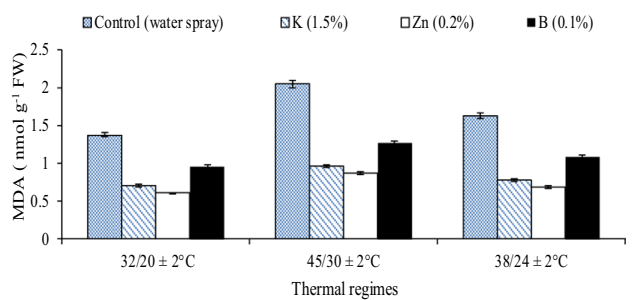

Figure 1. Effect of different thermal regimes and nutrients' spray on superoxide dismutase (SOD), catalase (CAT), peroxidase (POX U mg ${ }^{-1}$ protein), ascorbic acid (AsA $\mathrm{mg} \mathrm{g}^{-1} \mathrm{FW}$ ), total phenolic contents (TPC mg $\mathrm{g}^{-1} \mathrm{FW}$ ) and malondialdehyde contents (MDA nmol g${ }^{-1} \mathrm{FW}$ ), (averaged across of squaring, flowering and boll formation stages) of cotton leaves under glass house conditions.

\begin{tabular}{|l|l|l|l|l|l|l|l|}
\hline $\begin{array}{l}\text { Thermal } \\
\text { regimes }\end{array}$ & Nutrients & SOD & CAT & POX & ASA & TPC & MDA \\
\hline \multirow{5}{*}{$32 / 20^{\circ} \mathrm{C}$} & Control & $37.71 \mathrm{~d} \pm 2.0$ & $51.33 \mathrm{~d} \pm 3.13$ & $34.89 \mathrm{c} \pm 0.52$ & $137.92 \mathrm{~d} \pm 8.63$ & $4.63 \mathrm{~d} \pm 0.52$ & $1.37 \mathrm{a} \pm 0.65$ \\
\cline { 2 - 8 } & Potassium $(1.5 \%)$ & $46.31 \mathrm{~b} \pm 2.5$ & $91.85 \mathrm{~b} \pm 5.97$ & $47.96 \mathrm{~b} \pm 0.74$ & $195.58 \mathrm{~b} \pm 10.25$ & $6.06 \mathrm{~b} \pm 0.30$ & $0.70 \mathrm{c} \pm 0.40$ \\
\cline { 2 - 8 } & Zinc $(0.2 \%)$ & $56.71 \mathrm{a} \pm 3.0$ & $140.39 \mathrm{a} \pm 8.82$ & $50.96 \mathrm{a} \pm 0.98$ & $207.39 \mathrm{a} \pm 12.35$ & $7.27 \mathrm{a} \pm 0.41$ & $0.60 \mathrm{~d} \pm 0.35$ \\
\cline { 2 - 8 } & Boron $(0.1 \%)$ & $42.78 \mathrm{bc} \pm 2.3$ & $68.57 \mathrm{c} \pm 4.35$ & $35.23 \mathrm{c} \pm 0.56$ & $177.94 \mathrm{c} \pm 8.75$ & $5.18 \mathrm{c} \pm 0.25$ & $0.96 \mathrm{~b} \pm 0.48$ \\
\hline \multirow{5}{*}{$45 / 30^{\circ} \mathrm{C}$} & Control & $55.16 \mathrm{~d} \pm 2.8$ & $83.10 \mathrm{~d} \pm 4.54$ & $50.17 \mathrm{~d} \pm 0.78$ & $170.12 \mathrm{c} \pm 11.20$ & $8.00 \mathrm{c} \pm 0.41$ & $2.04 \mathrm{a} \pm 0.14$ \\
\cline { 2 - 8 } & Potassium $(1.5 \%)$ & $87.41 \mathrm{~b} \pm 5.1$ & $258.73 \mathrm{~b} \pm 13.65$ & $100.62 \mathrm{~b} \pm 1.5$ & $328.44 \mathrm{a} \pm 22.10$ & $14.25 \mathrm{~b} \pm 0.70$ & $0.96 \mathrm{c} \pm 0.051$ \\
\cline { 2 - 8 } & Zinc $(0.2 \%)$ & $111.83 \mathrm{a} \pm 6.2$ & $310.77 \mathrm{a} \pm 16.31$ & $104.79 \mathrm{a} \pm 1.3$ & $337.90 \mathrm{a} \pm 20.30$ & $15.23 \mathrm{a} \pm 0.84$ & $0.87 \mathrm{~d} \pm 0.044$ \\
\cline { 2 - 8 } & Boron $(0.1 \%)$ & $70.66 \mathrm{c} \pm 3.7$ & $189.87 \mathrm{c} \pm 10.19$ & $77.48 \mathrm{c} \pm 0.92$ & $250.51 \mathrm{~b} \pm 14.50$ & $10.38 \mathrm{~d} \pm 0.52$ & $1.26 \mathrm{~b} \pm 0.065$ \\
\hline \multirow{5}{*}{$38 / 24^{\circ} \mathrm{C}$} & Control & $47.23 \mathrm{~d} \pm 2.4$ & $66.17 \mathrm{~d} \pm 3.60$ & $41.70 \mathrm{~d} \pm 0.58$ & $156.32 \mathrm{c} \pm 8.4$ & $5.24 \mathrm{~d} \pm 0.32$ & $1.63 \mathrm{a} \pm 0.70$ \\
\cline { 2 - 9 } & Potassium $(1.5 \%)$ & $64.70 \mathrm{~b} \pm 3.5$ & $109.91 \mathrm{~b} \pm 6.63$ & $57.99 \mathrm{~b} \pm 0.71$ & $228.12 \mathrm{a} \pm 14.45$ & $7.48 \mathrm{~b} \pm 0.40$ & $0.77 \mathrm{c} \pm 0.50$ \\
\cline { 2 - 9 }$(0.2 \%)$ & $73.58 \mathrm{a} \pm 3.8$ & $160.17 \mathrm{a} \pm 9.22$ & $61.25 \mathrm{a} \pm 0.88$ & $230.39 \mathrm{a} \pm 16.76$ & $8.06 \mathrm{a} \pm 0.50$ & $0.69 \mathrm{~d} \pm 0.41$ \\
\cline { 2 - 9 } & Binc $(0.1 \%)$ & $51.29 \mathrm{c} \pm 2.8$ & $85.16 \mathrm{c} \pm 4.62$ & $48.71 \mathrm{c} \pm 0.64$ & $205.57 \mathrm{~b} \pm 11.20$ & $6.21 \mathrm{c} \pm 0.35$ & $1.08 \mathrm{~b} \pm 0.64$ \\
\cline { 2 - 8 } & LSD & 3.65 & 5.72 & 2.94 & 11.78 & 0.49 & \\
\hline
\end{tabular}

Table 2. Effect of different thermal regimes and nutrients' spray on superoxide dismutase (SOD), catalase

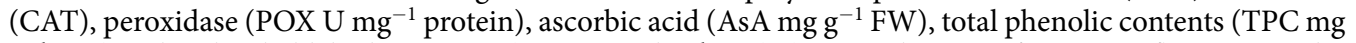
$\mathrm{g}^{-1} \mathrm{FW}$ ) and malondialdehyde contents (MDA nmol g ${ }^{-1} \mathrm{FW}$ ), (averaged across of squaring, flowering and boll formation stages) of cotton leaves under glass house conditions. Values are the means of three replications $(\mathrm{n}=4) \pm \mathrm{SE}$ and variants possessing the same letters are not statistically significant at $P<0.05$. Main factors and interaction are significant at $P<0.01$. Lettering is done separately for each thermal regime using the LSD of the interaction between thermal regimes and nutrients' spray. 
(a)

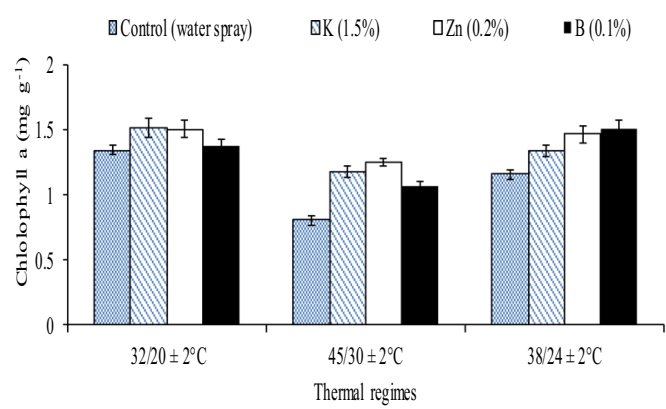

(c)

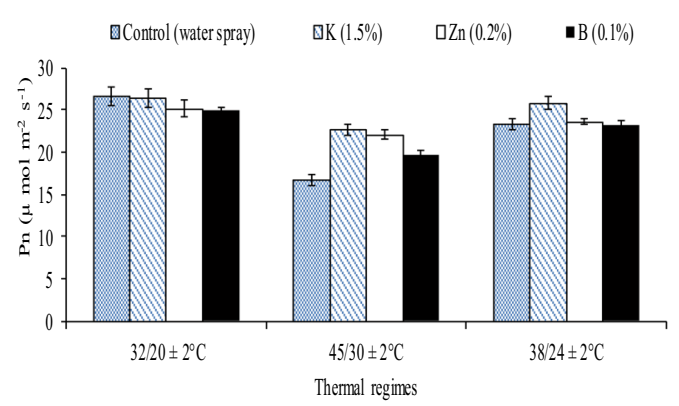

(e)

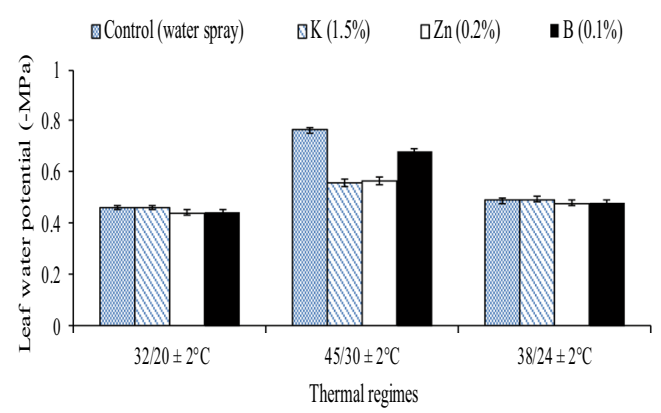

(b)

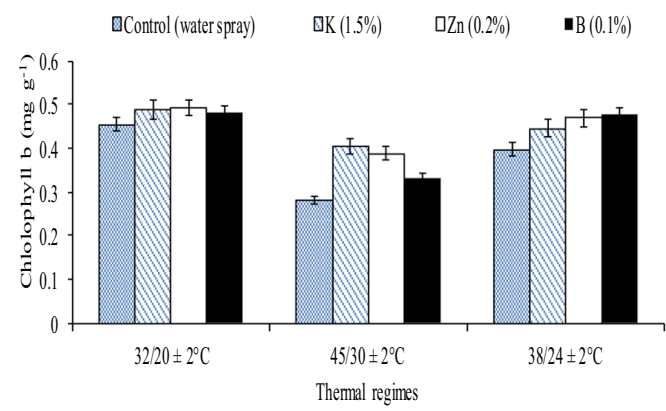

(d)

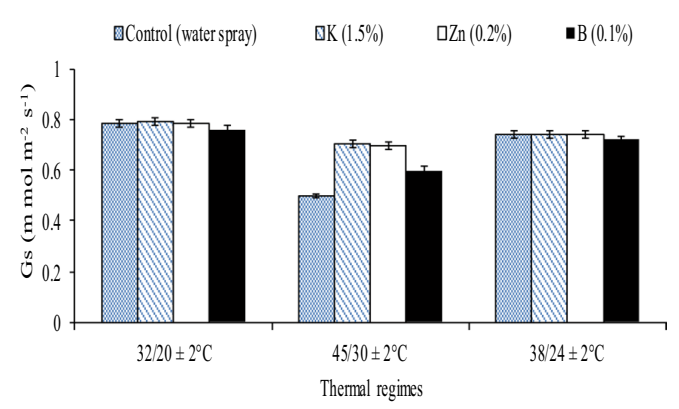

(f)

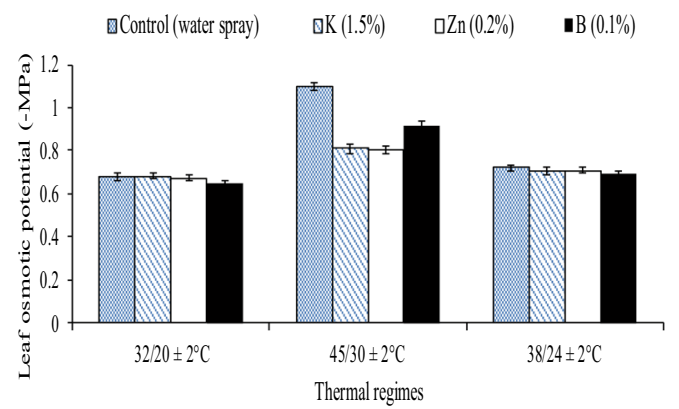

Figure 2. Effect of different thermal regimes and nutrients' spray on chlorophyll contents $(\mathrm{a}+\mathrm{b})(\mathrm{mg}$ $\left.\mathrm{g}^{-1} \mathrm{FW}\right)$, net photosynthetic rate-Pn $\left.\left(\mu \mathrm{mol} \mathrm{m}^{-2} \mathrm{sec}^{-1}\right), \mathrm{FW}\right)$, stomatal conductance $\left(\mathrm{Gs} \mathrm{m} \mathrm{mol} \mathrm{m}^{-2} \mathrm{~s}^{-1}\right)$, leaf water potential $(-\mathrm{MPa})$ and leaf osmotic potential $(-\mathrm{MPa})$ (averaged across of squaring, flowering and boll formation stages) of cotton leaves under glass house conditions.

not shown). All the plants were grown at $32 / 20^{\circ} \mathrm{C}$ up to $30 \mathrm{DAS}$ (before initiation of squaring). After that, pots were divided into 3 sets, each set was consisted of 20 pots which were transferred to growth chambers maintained for different temperature. First set of 20 pots was exposed to heat stress at squaring, $2^{\text {nd }}$ set at flowering and $3^{\text {rd }}$ set at boll formation. Heat stress was imposed for a period of one week at squaring, flowering and boll formation and data recorded were averaged across the three reproductive stages (squaring, flowering and boll formation). Samples from the youngest fully expanded leaves were collected immediately after removing the pots from stress, stored in liquid nitrogen and processed to record various attributes. The experiment was conducted using completely randomized design with split arrangement and replicated four times. Varying temperature regimes were imposed in main pots and nutrients were foliar applied in split pots.

Field experiment. The field experiments were conducted at Agronomic Research Area, University of Agriculture Faisalabad, Pakistan during 2012 to 2013. The meteorological data were collected by the Meteorological Observatory of the Department of Agronomy, UAF. Treatments were comprised of sowing dates in main plots viz. early April (medium temperature at squaring, flowering and boll formation), early May (high temperature at squaring, flowering and boll formation) and mid-June (optimum temperature at squaring, flowering and boll formation). While, split plot treatments were consisted of foliar sprays of $\mathrm{K}, \mathrm{Zn}$ and $\mathrm{B}$ viz. water spray, foliar spray of K@ 1.5\%, foliar spray of Zn @ 0.2\% and foliar spray of B @ 0.1\%. Different sowing times were 


\begin{tabular}{|l|l|l|l|l|l|l|l|}
\hline $\begin{array}{l}\text { Thermal } \\
\text { regimes }\end{array}$ & Nutrients & Chlorophyll a & Chlorophyll $\mathrm{b}$ & Pn & Gs & $\begin{array}{l}\text { Water } \\
\text { Potential }\end{array}$ & $\begin{array}{l}\text { Osmotic } \\
\text { potential }\end{array}$ \\
\hline \multirow{4}{*}{$32 / 20^{\circ} \mathrm{C}$} & Control & $1.34 \mathrm{~b} \pm 0.070$ & $0.46 \mathrm{ab} \pm 0.27$ & $26.69 \mathrm{a} \pm 0.63$ & $0.79 \mathrm{a} \pm 0.042$ & $0.46 \mathrm{a} \pm 0.041$ & $0.68 \mathrm{a} \pm 0.034$ \\
\cline { 2 - 8 } & Potassium $(1.5 \%)$ & $1.51 \mathrm{a} \pm 0.073$ & $0.48 \mathrm{a} \pm 0.30$ & $26.38 \mathrm{a} \pm 0.67$ & $0.79 \mathrm{a} \pm 0.040$ & $0.46 \mathrm{a} \pm 0.040$ & $0.68 \mathrm{a} \pm 0.032$ \\
\cline { 2 - 8 } & Zinc $(0.2 \%)$ & $1.50 \mathrm{a} \pm 0.076$ & $0.49 \mathrm{a} \pm 0.32$ & $26.20 \mathrm{a} \pm 0.57$ & $0.78 \mathrm{a} \pm 0.035$ & $0.44 \mathrm{a} \pm 0.039$ & $0.67 \mathrm{a} \pm 0.035$ \\
\cline { 2 - 8 } & Boron $(0.1 \%)$ & $1.37 \mathrm{~b} \pm 0.068$ & $0.48 \mathrm{a} \pm 0.26$ & $26.04 \mathrm{a} \pm 0.12$ & $0.77 \mathrm{a} \pm 0.036$ & $0.44 \mathrm{a} \pm 0.038$ & $0.66 \mathrm{a} \pm 0.031$ \\
\hline \multirow{3}{*}{$45 / 30^{\circ} \mathrm{C}$} & Control & $0.80 \mathrm{c} \pm 0.051$ & $0.28 \mathrm{c} \pm 0.20$ & $16.74 \mathrm{c} \pm 0.37$ & $0.50 \mathrm{c} \pm 0.024$ & $0.76 \mathrm{a} \pm 0.070$ & $1.10 \mathrm{a} \pm 0.52$ \\
\cline { 2 - 8 } & Potassium $(1.5 \%)$ & $1.18 \mathrm{a} \pm 0.062$ & $0.41 \mathrm{a} \pm 0.23$ & $22.66 \mathrm{a} \pm 0.40$ & $0.71 \mathrm{a} \pm 0.037$ & $0.56 \mathrm{c} \pm 0.052$ & $0.81 \mathrm{c} \pm 0.040$ \\
\cline { 2 - 8 } & Zinc $(0.2 \%)$ & $1.25 \mathrm{a} \pm 0.067$ & $0.39 \mathrm{a} \pm 0.21$ & $22.11 \mathrm{a} \pm 0.37$ & $0.70 \mathrm{a} \pm 0.033$ & $0.57 \mathrm{c} \pm 0.055$ & $0.80 \mathrm{c} \pm 0.038$ \\
\cline { 2 - 8 } & Boron $(0.1 \%)$ & $1.07 \mathrm{~b} \pm 0.055$ & $0.33 \mathrm{~b} \pm 0.19$ & $19.79 \mathrm{~b} \pm 0.29$ & $0.60 \mathrm{~b} \pm 0.031$ & $0.68 \mathrm{~b} \pm 0.064$ & $0.91 \mathrm{~b} \pm 0.045$ \\
\hline \multirow{5}{*}{$38 / 24^{\circ} \mathrm{C}$} & Control & $1.16 \mathrm{c} \pm 0.059$ & $0.40 \mathrm{c} \pm 0.22$ & $22.29 \mathrm{~b} \pm 0.35$ & $0.74 \mathrm{a} \pm 0.034$ & $0.49 \mathrm{a} \pm 0.044$ & $0.72 \mathrm{a} \pm 0.030$ \\
\cline { 2 - 8 } & Potassium $(1.5 \%)$ & $1.33 \mathrm{~b} \pm 0.063$ & $0.45 \mathrm{ab} \pm 0.25$ & $25.55 \mathrm{a} \pm 0.49$ & $0.74 \mathrm{a} \pm 0.037$ & $0.49 \mathrm{a} \pm 0.045$ & $0.71 \mathrm{a} \pm 0.034$ \\
\cline { 2 - 8 } & Zinc $(0.2 \%)$ & $1.46 \mathrm{a} \pm 0.079$ & $0.47 \mathrm{a} \pm 0.28$ & $25.33 \mathrm{a} \pm 0.16$ & $0.74 \mathrm{a} \pm 0.038$ & $0.48 \mathrm{a} \pm 0.043$ & $0.71 \mathrm{a} \pm 0.035$ \\
\cline { 2 - 8 } & Boron $(0.1 \%)$ & $1.50 \mathrm{a} \pm 0.086$ & $0.48 \mathrm{a} \pm 0.31$ & $23.87 \mathrm{~b} \pm 0.37$ & $0.72 \mathrm{a} \pm 0.033$ & $0.48 \mathrm{a} \pm 0.046$ & $0.70 \mathrm{a} \pm 0.035$ \\
\cline { 2 - 8 } & LSD & 0.080 & 0.023 & 1.38 & 0.032 & 0.021 & 0.031 \\
\hline
\end{tabular}

Table 3. Effect of different thermal regimes and nutrients' spray on chlorophyll contents $(\mathrm{a}+\mathrm{b})\left(\mathrm{mg} \mathrm{g}^{-1} \mathrm{FW}\right)$, net photosynthetic rate- $\left.\mathrm{Pn}\left(\mu \mathrm{mol} \mathrm{m} \mathrm{m}^{-2} \mathrm{sec}^{-1}\right), \mathrm{FW}\right)$, stomatal conductance $\left(\mathrm{Gs} \mathrm{m} \mathrm{mol} \mathrm{m} \mathrm{m}^{-1}\right.$ ), leaf water potential $(-\mathrm{MPa})$ and leaf osmotic potential $(-\mathrm{MPa})$ (averaged across of squaring, flowering and boll formation stages) of cotton leaves under glass house conditions. Values are the means of three replications $(\mathrm{n}=4) \pm \mathrm{SE}$ and variants possessing the same letters are not statistically significant at $P<0.05$. Main factors and interaction are significant at $P<0.01$. Lettering is done separately for each thermal regime using the LSD of the interaction between thermal regimes and nutrients' spray.

\begin{tabular}{|l|l|l|l|}
\hline $\begin{array}{l}\text { Thermal } \\
\text { regimes }\end{array}$ & Nutrients & Boll Weight $(\mathrm{g})$ & SCY \\
\hline \multirow{5}{*}{$32 / 20^{\circ} \mathrm{C}$} & Control & $3.70 \mathrm{c} \pm 0.15$ & $83.22 \mathrm{a} \pm 3.1$ \\
\cline { 2 - 4 } & Potassium $(1.5 \%)$ & $4.36 \mathrm{a} \pm 0.22$ & $84.99 \mathrm{a} \pm 2.9$ \\
\cline { 2 - 4 } & Zinc $(0.2 \%)$ & $4.25 \mathrm{a} \pm 0.20$ & $85.05 \mathrm{a} \pm 4.1$ \\
\cline { 2 - 4 } & Boron $(0.1 \%)$ & $4.13 \mathrm{ab} \pm 0.18$ & $85.47 \mathrm{a} \pm 4.2$ \\
\hline \multirow{5}{*}{$45 / 30^{\circ} \mathrm{C}$} & Control & $2.96 \mathrm{~b} \pm 0.14$ & $49.99 \mathrm{c} \pm 2.7$ \\
\cline { 2 - 4 } & Potassium $(1.5 \%)$ & $3.59 \mathrm{a} \pm 0.22$ & $60.80 \mathrm{a} \pm 3.3$ \\
\cline { 2 - 4 } & Zinc $(0.2 \%)$ & $3.54 \mathrm{a} \pm 0.16$ & $58.17 \mathrm{a} \pm 2.8$ \\
\cline { 2 - 4 } & Boron $(0.1 \%)$ & $3.54 \mathrm{a} \pm 0.17$ & $53.67 \mathrm{~b} \pm 2.7$ \\
\hline $38 / 24^{\circ} \mathrm{C}$ & Control & $3.38 \mathrm{~b} \pm 0.11$ & $67.69 \mathrm{c} \pm 4.4$ \\
\cline { 2 - 4 } & Potassium $(1.5 \%)$ & $3.70 \mathrm{a} \pm 0.19$ & $77.45 \mathrm{a} \pm 4.0$ \\
\cline { 2 - 4 } & Zinc $(0.2 \%)$ & $3.75 \mathrm{a} \pm 0.15$ & $75.92 \mathrm{a} \pm 3.8$ \\
\cline { 2 - 4 } & Boron $(0.1 \%)$ & $3.73 \mathrm{a} \pm 0.14$ & $71.34 \mathrm{~b} \pm 3.7$ \\
\cline { 2 - 4 } & LSD & 0.20 & 2.99 \\
\hline
\end{tabular}

Table 4. Effect of different thermal regimes and nutrients' spray on averaged boll weight (g) and seed cotton yield per plant $(\mathrm{g})$ of cotton crop under glass house conditions. Values are the means of three replications $(\mathrm{n}=4) \pm \mathrm{SE}$ and variants possessing the same letters are not statistically significant at $P<0.05$. Main factors and interaction are significant at $P<0.01$. Lettering is done separately for each thermal regime using the LSD of the interaction between thermal

referred as thermal regimes. The varying temperature was recorded at squaring, flowering and boll formation stages. Three sowing times (April 2, May 3 and June 17 during 2012 and April 4, May 2 and June 19 during 2013) were selected based on previous five years' climate data. Sowing of both experiments was done on sandy clay loam soil at times as per treatments during 2012 and 2013. Seed of cotton variety (AA- 802) was collected from Ali Akbar Enterprises during both years of study. Crop was planted with manual dibbling having $75 \mathrm{~cm}$ apart ridges and plant to plant distance was $30 \mathrm{~cm}$. Weeds were controlled by two hoeing i.e. at squaring ( 35 days after planting) and at flowering (60 days after planting) while the sucking insects and boll worms were controlled with insecticides. Nine irrigations were applied as per crop requirement keeping in view the reproductive stages having heat stress at different sowing dates to avoid the drought stress during heat stress periods. The data of physiological parameters were recorded across the three reproductive stages of cotton i.e. squaring, flowering and boll formation. June thermal regime (late sown crop) was considered control, as it provided optimal temperature at all reproductive stages, while April (early sowing) and May sown crops were experienced sub and supra-optimal temperatures at three reproductive stages (Table 1). The experiment was laid out in randomized complete design with split treatment structure having three replications. Sowing dates were randomized in main and exogenous nutrients in split plots. 
$(2)$

(b)

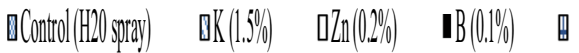
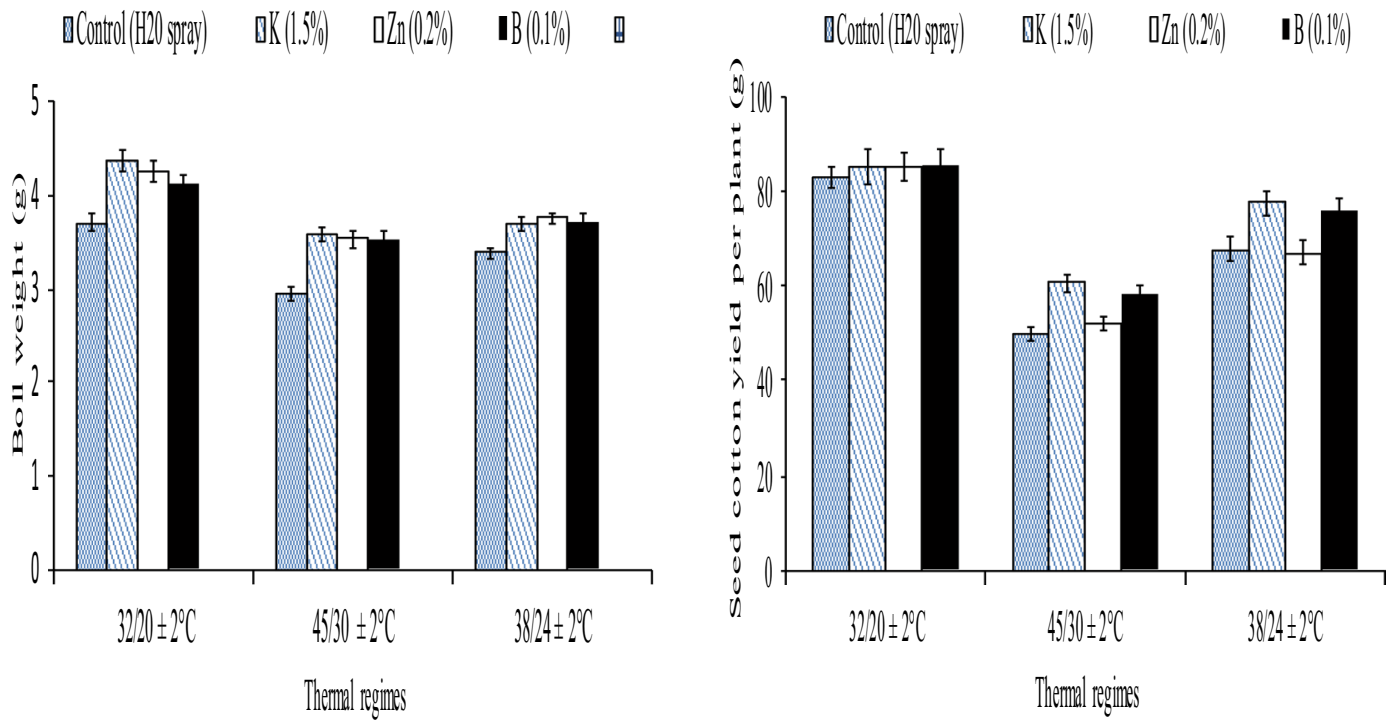

Figure 3. Effect of different thermal regimes and nutrients' spray on averaged boll weight ( $\mathrm{g}$ ) and seed cotton yield per plant $(\mathrm{g})$ of cotton crop under glass house conditions.

\begin{tabular}{|c|c|c|c|c|c|c|c|c|c|}
\hline $\begin{array}{l}\text { Thermal } \\
\text { regimes }\end{array}$ & Nutrients & SOD 2012 & SOD 2013 & CAT 2012 & CAT 2013 & POX 2012 & \begin{tabular}{|l|} 
POX \\
2013
\end{tabular} & AsA 2012 & AsA 2013 \\
\hline \multirow{4}{*}{$\begin{array}{l}\text { Optimal } \\
\text { regimes of } \\
\text { sowing dates }\end{array}$} & Control & $41.33 \mathrm{~cd} \pm 3.8$ & $39.42 b c \pm 3.5$ & $58.11 \mathrm{~cd} \pm 5.60$ & $59.49 \mathrm{~cd} \pm 5.60$ & $\begin{array}{l}37.35 \\
a b \pm 3.6\end{array}$ & $\begin{array}{l}35.30 \\
b \pm 3.0\end{array}$ & $\begin{array}{l}147.94 \\
b \pm 13.40\end{array}$ & $\begin{array}{l}145.45 \\
\mathrm{~b} \pm 14.0\end{array}$ \\
\hline & Potassium (1.5\%) & $47.55 \mathrm{~b} \pm 4.4$ & $47.69 \mathrm{a} \pm 4.3$ & $88.01 \mathrm{~b} \pm 7.08$ & $93.48 \mathrm{~b} \pm 7.08$ & $\begin{array}{l}45.04 \\
a \pm 3.9\end{array}$ & $\begin{array}{l}49.49 \\
\mathrm{a} \pm 4.6\end{array}$ & $\begin{array}{l}204.21 \\
\mathrm{a} \pm 18.70\end{array}$ & $\begin{array}{l}196.40 \\
\mathrm{a} \pm 17.0\end{array}$ \\
\hline & Zinc $(0.2 \%)$ & $58.73 \mathrm{a} \pm 5.3$ & $57.35 \mathrm{a} \pm 5.1$ & $140.86 \mathrm{a} \pm 10.70$ & $144.94 \mathrm{a} \pm 10.70$ & $\begin{array}{l}47.62 \\
\mathrm{a} \pm 4.1\end{array}$ & $\begin{array}{l}52.47 \\
\mathrm{a} \pm 4.5\end{array}$ & $\begin{array}{l}215.12 \\
\mathrm{a} \pm 19.40\end{array}$ & $\begin{array}{l}206.47 \\
\mathrm{a} \pm 19.6\end{array}$ \\
\hline & Boron $(0.1 \%)$ & $44.10 \mathrm{bc} \pm 4.1$ & $43.12 \mathrm{ab} \pm 3.7$ & $73.11 \mathrm{bc} \pm 5.60$ & $76.42 \mathrm{bc} \pm 5.60$ & $\begin{array}{l}39.67 \\
a \pm 3.7\end{array}$ & $\begin{array}{l}38.07 \\
b \pm 3.6\end{array}$ & $\begin{array}{l}192.34 \\
\mathrm{a} \pm 17.40\end{array}$ & $\begin{array}{l}187.12 \\
\mathrm{a} \pm 18.20\end{array}$ \\
\hline \multirow{4}{*}{$\begin{array}{l}\text { Supra- } \\
\text { optimal of } \\
\text { sowing dates }\end{array}$} & Control & $56.04 \mathrm{~d} \pm 4.9$ & $54.04 \mathrm{~d} \pm 4.9$ & $81.81 \mathrm{~d} \pm 5.90$ & $78.22 \mathrm{~d} \pm 5.90$ & $\begin{array}{l}46.86 \\
c \pm 4.0\end{array}$ & $\begin{array}{l}41.85 \\
c \pm 4.0\end{array}$ & $\begin{array}{l}164.57 \\
c \pm 15.8\end{array}$ & $\begin{array}{l}158.87 \\
c \pm 14.50\end{array}$ \\
\hline & Potassium (1.5\%) & $86.66 \mathrm{~b} \pm 7.8$ & $84.34 \mathrm{~b} \pm 8.1$ & $230.87 \mathrm{~b} \pm 10.30$ & $221.95 b \pm 10.30$ & $\begin{array}{l}93.14 \\
\mathrm{a} \pm 8.3\end{array}$ & $\begin{array}{l}86.44 \\
a \pm 7.6\end{array}$ & $\begin{array}{l}312.83 \\
\mathrm{a} \pm 30.20\end{array}$ & $\begin{array}{l}298.17 \\
\mathrm{a} \pm 27.60\end{array}$ \\
\hline & Zinc $(0.2 \%)$ & $110.28 \mathrm{a} \pm 10.1$ & $105.12 \mathrm{a} \pm 9.9$ & $280.19 a \pm 10.66$ & $264.68 \mathrm{a} \pm 10.66$ & $\begin{array}{l}101.56 \\
\mathrm{a} \pm 9.7\end{array}$ & $\begin{array}{l}91.34 \\
\mathrm{a} \pm 8.2\end{array}$ & $\begin{array}{l}325.01 \\
\mathrm{a} \pm 31.50\end{array}$ & $\begin{array}{l}310.61 \\
\mathrm{a} \pm 30.14\end{array}$ \\
\hline & Boron $(0.1 \%)$ & $69.17 c \pm 6.3$ & $66.75 c \pm 6.1$ & $161.45 c \pm 4.07$ & $153.64 \mathrm{c} \pm 4.07$ & $\begin{array}{l}74.61 \\
b \pm 6.6\end{array}$ & $\begin{array}{l}71.32 \\
b \pm 6.5\end{array}$ & $\begin{array}{l}239.03 \\
b \pm 22.60\end{array}$ & $\begin{array}{l}221.04 \\
b \pm 20.90\end{array}$ \\
\hline \multirow{5}{*}{$\begin{array}{l}\text { Sub-optimal } \\
\text { of sowing } \\
\text { dates }\end{array}$} & Control & $46.21 \mathrm{~cd} \pm 4.3$ & $43.79 \mathrm{~cd} \pm 3.6$ & $73.17 \mathrm{~d} \pm 5.47$ & $70.24 \mathrm{~cd} \pm 5.47$ & $\begin{array}{l}41.89 \\
\mathrm{bc} \pm 4.0\end{array}$ & $\begin{array}{l}38.08 \\
c \pm 3.2\end{array}$ & $\begin{array}{l}154.05 \\
\mathrm{~b} \pm 14.80\end{array}$ & $\begin{array}{l}155.11 \\
b \pm 13.50\end{array}$ \\
\hline & Potassium (1.5\%) & $56.54 \mathrm{~b} \pm 5.1$ & $52.63 \mathrm{~b} \pm 4.9$ & $148.08 \mathrm{~b} \pm 6.53$ & $122.20 \mathrm{~b} \pm 6.53$ & $\begin{array}{l}62.00 \\
\mathrm{a} \pm 5.2\end{array}$ & $\begin{array}{l}67.92 \\
a \pm 5.4\end{array}$ & $\begin{array}{l}221.52 \\
\mathrm{a} \pm 21.40\end{array}$ & $\begin{array}{l}211.13 \\
\mathrm{a} \pm 21.30\end{array}$ \\
\hline & Zinc $(0.2 \%)$ & $67.04 \mathrm{a} \pm 6.5$ & $64.42 \mathrm{a} \pm 6.2$ & $192.29 \mathrm{a} \pm 11.47$ & $168.10 \mathrm{a} \pm 11.47$ & $\begin{array}{l}65.23 \\
a \pm 5.8\end{array}$ & $\begin{array}{l}72.38 \\
\mathrm{a} \pm 6.5\end{array}$ & $\begin{array}{l}225.64 \\
\mathrm{a} \pm 21.0\end{array}$ & $\begin{array}{l}223.88 \\
\mathrm{a} \pm 20.80\end{array}$ \\
\hline & Boron $(0.1 \%)$ & $51.56 \mathrm{bc} \pm 4.5$ & $49.21 \mathrm{bc} \pm 4.8$ & $112.44 \mathrm{c} \pm 7.17$ & $95.41 \mathrm{c} \pm 7.17$ & $\begin{array}{l}50.43 \\
\mathrm{~b} \pm 4.6\end{array}$ & $\begin{array}{l}51.00 \\
\mathrm{~b} \pm 4.2\end{array}$ & $\begin{array}{l}201.08 \\
\mathrm{a} \pm 18.40\end{array}$ & $\begin{array}{l}200.43 \\
\mathrm{a} \pm 17.80\end{array}$ \\
\hline & LSD & 10.46 & 11.04 & 27.83 & 25.86 & 11.00 & 7.80 & 37.93 & 33.19 \\
\hline
\end{tabular}

Table 5. Effect of different thermal regimes and nutrients' spray on superoxide dismutase (SOD), catalase (CAT), peroxidase (POX U mg ${ }^{-1}$ protein) and ascorbic acid (AsA mg g ${ }^{-1} \mathrm{FW}$ ) contents (averaged across of squaring, flowering and boll formation stages) of cotton leaves under field conditions during 2012 and 2013. Values are the means of three replications $(n=3) \pm S E$ and variants possessing the same letters are not statistically significant at $P<0.05$. Lettering is done separately for each thermal regime using the LSD of the interaction between thermal regimes and nutrients' spray. 
(a)

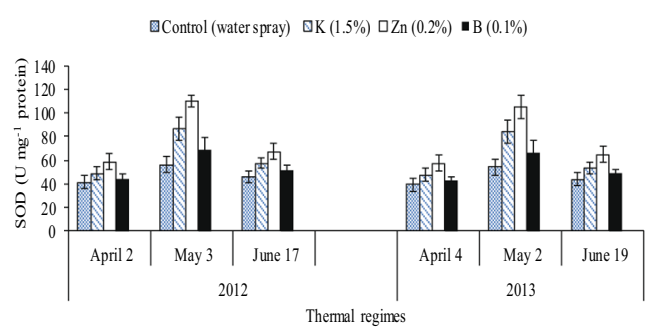

(c)

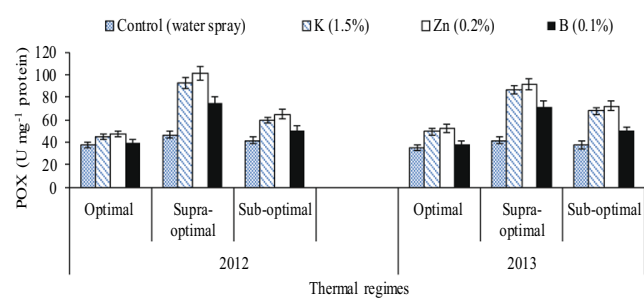

(e)

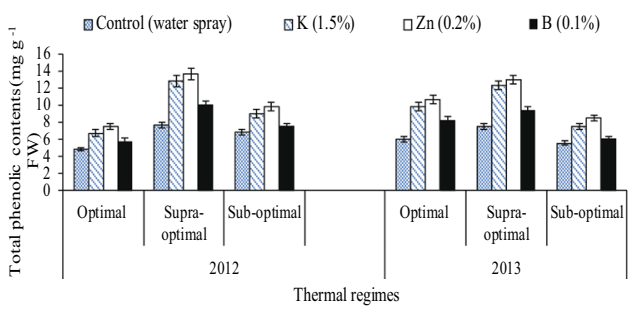

(b)

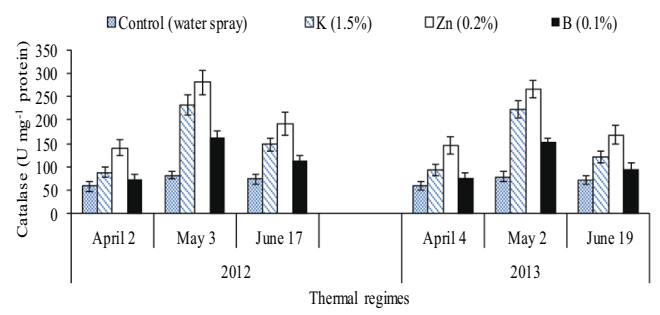

(d)

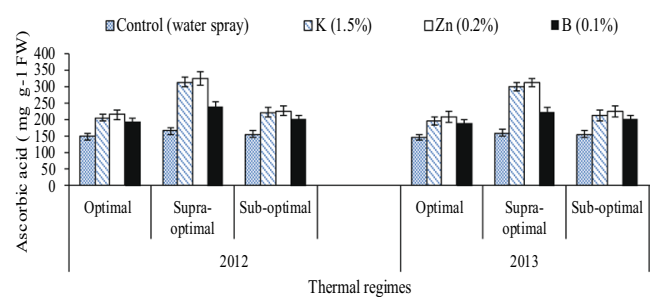

(f)

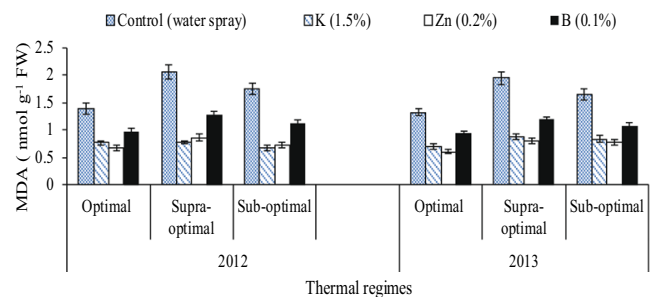

Figure 4. Effect of different thermal regimes and nutrients' spray on effect of different thermal regimes and nutrients' spray on superoxide dismutase (SOD), catalase (CAT), peroxidase (POX U mg ${ }^{-1}$ protein), ascorbic acid (AsA mg g ${ }^{-1} \mathrm{FW}$ ), total phenolic contents (TPC $\mathrm{mg} \mathrm{g}^{-1} \mathrm{FW}$ ) and malondialdehyde contents (MDA $\mathrm{nmol}{ }^{-1} \mathrm{FW}$ ), (averaged across of squaring, flowering and boll formation stages) of cotton leaves under field conditions during 2012 and 2013.

\begin{tabular}{|c|c|c|c|c|c|c|c|c|c|}
\hline $\begin{array}{l}\text { Thermal } \\
\text { regimes }\end{array}$ & Nutrients & TPC 2012 & TPC 2013 & MDA 2012 & MDA 2013 & $\begin{array}{l}\text { Chlorophyll } \\
\text { a } 2012\end{array}$ & $\begin{array}{l}\text { Chlorophyll } \\
\text { a } 2013\end{array}$ & $\begin{array}{l}\text { Chlorophyll } \\
\text { b } 2012\end{array}$ & $\begin{array}{l}\text { Chlorophyll } \\
\text { b } 2013\end{array}$ \\
\hline \multirow{4}{*}{$\begin{array}{l}\text { Optimal } \\
\text { regimes } \\
\text { of sowing } \\
\text { dates }\end{array}$} & Control & $4.86 \mathrm{~b} \pm 0.37$ & $5.98 c \pm 0.45$ & $1.39 \mathrm{a} \pm 0.87$ & $1.32 \mathrm{a} \pm 0.97$ & $1.48 \mathrm{ab} \pm 0.11$ & $1.42 \mathrm{a} \pm 0.12$ & $0.48 \mathrm{ab} \pm 0.39$ & $0.50 \mathrm{a} \pm 0.42$ \\
\hline & Potassium (1.5\%) & $6.40 \mathrm{a} \pm 0.52$ & $9.82 \mathrm{a} \pm 0.80$ & $0.76 \mathrm{c} \pm 0.065$ & $0.70 c \pm 0.62$ & $1.62 \mathrm{a} \pm 0.13$ & $1.50 \mathrm{a} \pm 0.11$ & $0.52 \mathrm{a} \pm 0.45$ & $0.54 \mathrm{a} \pm 0.50$ \\
\hline & Zinc (0.2\%) & $7.40 \mathrm{a} \pm 0.60$ & $10.60 \mathrm{a} \pm 0.91$ & $0.66 \mathrm{c} \pm 0.052$ & $0.60 c \pm 0.48$ & $1.56 \mathrm{a} \pm 0.10$ & $1.52 \mathrm{a} \pm 0.13$ & $0.53 \mathrm{a} \pm 0.47$ & $0.54 \mathrm{a} \pm 0.48$ \\
\hline & Boron $(0.1 \%)$ & $5.81 \mathrm{~b} \pm 0.48$ & $8.13 \mathrm{~b} \pm 0.72$ & $0.97 \mathrm{~b} \pm 0.078$ & $0.93 \mathrm{~b} \pm 0.84$ & $1.47 \mathrm{ab} \pm 0.12$ & $1.38 \mathrm{a} \pm 0.09$ & $0.52 \mathrm{a} \pm 0.50$ & $0.50 \mathrm{a} \pm 0.35$ \\
\hline \multirow{4}{*}{$\begin{array}{l}\text { Supra- } \\
\text { optimal } \\
\text { of sowing } \\
\text { dates }\end{array}$} & Control & $7.65 c \pm 0.55$ & $7.43 c \pm 0.60$ & $2.06 \mathrm{a} \pm 0.60$ & $1.94 \mathrm{a} \pm 0.17$ & $0.88 c \pm 0.072$ & $0.92 c \pm 0.07$ & $0.31 c \pm 0.26$ & $0.33 c \pm 0.27$ \\
\hline & Potassium (1.5\%) & $12.82 \mathrm{a} \pm 1.0$ & $12.23 \mathrm{a} \pm 0.99$ & $0.76 c \pm 0.06$ & $0.88 c \pm 0.74$ & $1.25 \mathrm{a} \pm 0.10$ & $1.30 \mathrm{a} \pm 0.11$ & $0.43 \mathrm{a} \pm 0.40$ & $0.45 \mathrm{a} \pm 0.32$ \\
\hline & Zinc (0.2\%) & $13.60 \mathrm{a} \pm 1.1$ & $12.95 \mathrm{a} \pm 1.1$ & $0.86 \mathrm{c} \pm 0.074$ & $0.80 \mathrm{c} \pm 0.70$ & $1.34 \mathrm{a} \pm 0.12$ & $1.37 \mathrm{a} \pm 0.10$ & $0.43 \mathrm{a} \pm 0.38$ & $0.49 \mathrm{a} \pm 0.30$ \\
\hline & Boron $(0.1 \%)$ & $9.97 \mathrm{~b} \pm 0.80$ & $9.29 \mathrm{~b} \pm 0.80$ & $1.28 \mathrm{~b} \pm 0.94$ & $1.19 \mathrm{~b} \pm 0.10$ & $\begin{array}{l}1.18 \\
\mathrm{ab} \pm 0.091\end{array}$ & $1.21 \mathrm{ab} \pm 0.08$ & $0.36 \mathrm{~b} \pm 0.30$ & $0.39 \mathrm{~b} \pm 0.25$ \\
\hline \multirow{5}{*}{$\begin{array}{l}\text { Sub- } \\
\text { optimal } \\
\text { of sowing } \\
\text { dates }\end{array}$} & Control & $6.89 c \pm 0.56$ & $5.55 c \pm 0.49$ & $1.74 \mathrm{a} \pm 1.30$ & $1.65 \mathrm{a} \pm 0.49$ & $1.28 \mathrm{ab} \pm 0.11$ & $1.26 \mathrm{~b} \pm 0.09$ & $0.42 \mathrm{bc} \pm 0.41$ & $0.44 \mathrm{ab} \pm 0.36$ \\
\hline & Potassium (1.5\%) & $8.93 \mathrm{a} \pm 0.75$ & $7.53 \mathrm{a} \pm 0.62$ & $0.67 c \pm 0.62$ & $0.83 \mathrm{c} \pm 0.77$ & $1.37 \mathrm{a} \pm 0.10$ & $1.41 \mathrm{a} \pm 0.11$ & $0.47 \mathrm{a} \pm 0.45$ & $0.50 \mathrm{a} \pm 0.39$ \\
\hline & Zinc (0.2\%) & $9.80 \mathrm{a} \pm 0.85$ & $8.42 \mathrm{a} \pm 0.72$ & $0.72 c \pm 0.68$ & $0.76 \mathrm{c} \pm 0.66$ & $1.41 \mathrm{a} \pm 0.13$ & $1.47 \mathrm{a} \pm 0.13$ & $0.47 \mathrm{a} \pm 0.44$ & $0.48 \mathrm{a} \pm 0.41$ \\
\hline & Boron $(0.1 \%)$ & $7.50 \mathrm{~b} \pm 0.68$ & $6.05 \mathrm{~b} \pm 0.55$ & $1.13 \mathrm{~b} \pm 0.93$ & $1.07 \mathrm{~b} \pm 0.10$ & $1.44 \mathrm{a} \pm 0.12$ & $1.53 \mathrm{a} \pm 0.14$ & $0.46 \mathrm{ab} \pm 0.30$ & $0.48 \mathrm{a} \pm 0.38$ \\
\hline & LSD & 1.37 & 0.88 & 0.18 & 0.11 & 0.13 & 0.16 & 0.040 & 0.050 \\
\hline
\end{tabular}

Table 6. Effect of different thermal regimes and nutrients' spray on total phenolic contents (TPC $\mathrm{mg} \mathrm{g}^{-1} \mathrm{FW}$ ), malondialdehyde contents (MDA nmol g $\left.\mathrm{g}^{-1} \mathrm{FW}\right)$ and chlorophyll contents $(\mathrm{a}+\mathrm{b})\left(\mathrm{mg} \mathrm{g}^{-1} \mathrm{FW}\right)$ (averaged across of squaring, flowering and boll formation stages) of cotton leaves under field conditions during 2012 and 2013. Values are the means of three replications $(n=3) \pm S E$ and variants possessing the same letters are not statistically significant at $P^{<} 0.05$. Lettering is done separately for each thermal regime using the LSD of the interaction between thermal regimes and nutrients' spray. 
(a)

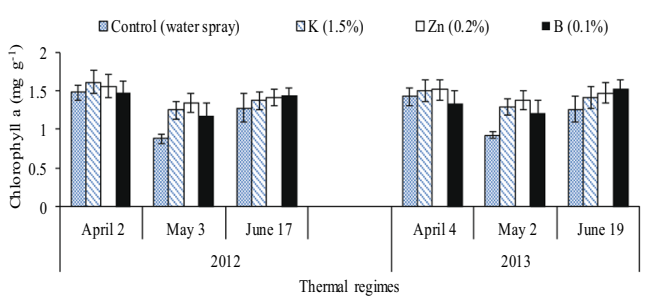

(c)

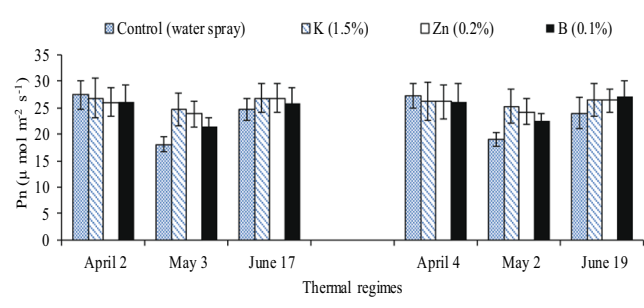

(e)

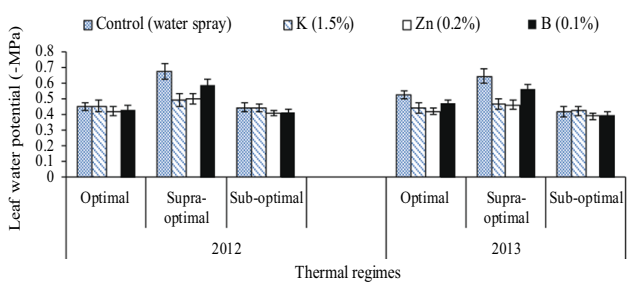

(b)

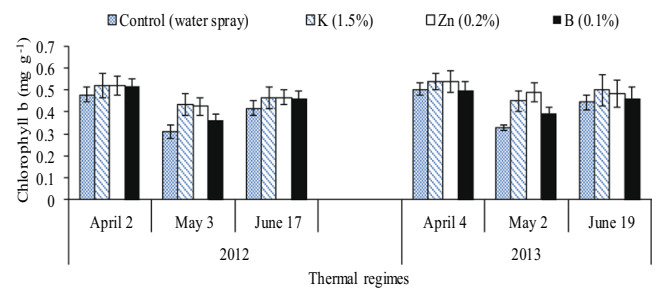

(d)

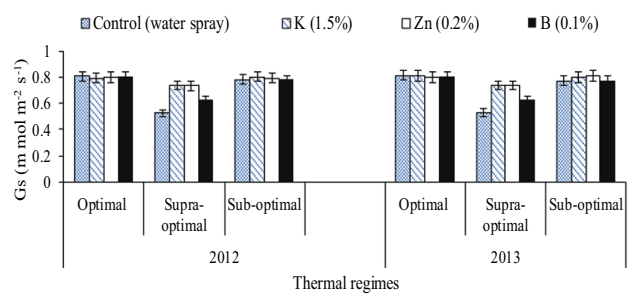

(f)

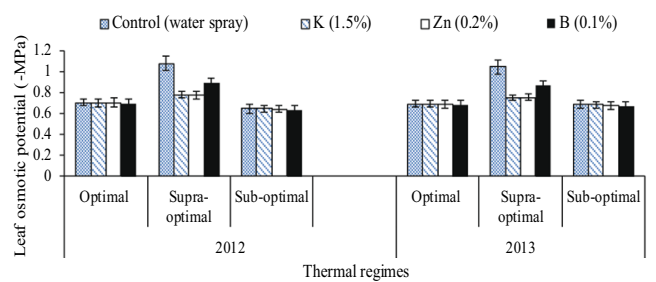

Figure 5. Effect of different thermal regimes and nutrients' spray on chlorophyll contents $(\mathrm{a}+\mathrm{b})(\mathrm{mg}$ $\left.\mathrm{g}^{-1} \mathrm{FW}\right)$, net photosynthetic rate-Pn $\left.\left(\mu \mathrm{mol} \mathrm{m}^{-2} \mathrm{sec}^{-1}\right), \mathrm{FW}\right)$, stomatal conductance $\left(\mathrm{Gs} \mathrm{m} \mathrm{mol} \mathrm{m} \mathrm{m} \mathrm{s}^{-1}\right)$, leaf water potential $(-\mathrm{MPa})$ and leaf osmotic potential $(-\mathrm{MPa})$ (averaged across of squaring, flowering and boll formation stages) of cotton leaves during 2012 and 2013.

Observations. Biochemical assays. Leaves samples weighing $0.5 \mathrm{~g}$ were extracted in with $10 \mathrm{ml}$ of phosphate buffer ( $\mathrm{pH}$ 7.8) for the extraction of enzymes. The supernatant was used for enzyme determination after centrifuge and the residues were discarded. The extracted material was stored at $4{ }^{\circ} \mathrm{C}^{27}$. The samples for all enzymatic and non-enzymatic antioxidants were pipetted into 96 -well plates. The plates were, then, read by micro plate reader (ELX800, Bio-Tek Instruments, Inc., Winooski, VT, USA) at different wavelengths. Superoxide dismutase contents were determined by ${ }^{28}$ method. Superoxide dismutase was quantified as enzymes units that inhibited photo reduction of nitrobluetetrazolium (NBT) and recorded the absorbance at $470 \mathrm{~nm}$. While, CAT was measured as enzymes units that converted $\mathrm{H}_{2} \mathrm{O}_{2}$ to $\mathrm{H}_{2} \mathrm{O}$ and $\mathrm{O}_{2}$ using the protocol as described by $\mathrm{Liu}^{29}$. The reaction mixture $\left[50 \mathrm{mM}\right.$ phosphate buffer $(\mathrm{pH} 7)+5.9 \mathrm{mM} \mathrm{H}_{2} \mathrm{O}_{2}$ ] was mixed with $0.1 \mathrm{~mL}$ enzyme extract and read the absorbance at $240 \mathrm{~nm}$. Peroxidase contents were determined using method as given by ${ }^{29}$. Peroxidase was quantified as units of enzymes that oxidized guaiacol. The reaction mixture was comprised of $50 \mathrm{mM}$ phosphate buffer $(\mathrm{pH} 5)+40 \mathrm{mM} \mathrm{H}_{2} \mathrm{O}_{2}+20 \mathrm{mM}$ guaiacol and $0.1 \mathrm{~mL}$ of enzyme extract per each sample. The absorbance was recorded at wavelength of $470 \mathrm{~nm}$.

For the estimation of ascorbic acid, $900 \mu \mathrm{L}$ dist. $\mathrm{H}_{2} \mathrm{O}+100 \mu \mathrm{L}$ sample extract $+1 \mathrm{~mL}$ dichlorophenol-indophenol $+100 \mu \mathrm{L} 0.1 \%$ Meta $\mathrm{H}_{3} \mathrm{PO}_{4}$ were mixed in a test tube and absorbance was recorded at $520 \mathrm{~nm}^{30}$. Folin-Ciocalteu (FC) reagent method was used for the determination of TPC ${ }^{31}$. Leaves samples of $0.5 \mathrm{~g}$ weight were extracts with $80 \%$ acetone $(10 \mathrm{~mL})$ and centrifuged. Enzyme extract $(20 \mu \mathrm{L})+\mathrm{FC}$-reagent $(100 \mu \mathrm{L})+1.5 \mathrm{~mL}$ water were mixed in a cuvette and placed for 30 minutes. Then, added $700 \mathrm{mM} \mathrm{Na}_{2} \mathrm{CO}_{3}$ and incubated at room temperature for period of 2 hours. The absorbance was taken at $765 \mathrm{~nm}$ having $200 \mu \mathrm{L}$ sample in each well. MDA contents in cotton leaves were determined according following the procedure as adapted by ${ }^{32}$. Leaf sample $(0.5 \mathrm{~g})$ was homogenized in $10 \mathrm{ml}$ of $0.1 \%$ trichloroacetic acid (TCA) solution and centrifuged at $12000 \times \mathrm{g}$ for 15 minutes. For each $\mathrm{mL}$ of extract $4.5 \mathrm{ml}$ of thiobarbituric acid $(0.5 \%)$ was used with the reaction mixture and heated at $95^{\circ} \mathrm{C}$ for $30 \mathrm{~min}$ and cooled. The absorbance was taken at 532 and $600 \mathrm{~nm}$ and MDA concentration was determine using formula:

$$
\text { MDA level }(\mathrm{nmol})=\frac{\Delta(\mathrm{A} 532 \mathrm{~nm}-\mathrm{A} 600 \mathrm{~nm})}{156 \times 10^{5}}
$$

$A=$ Absorption coefficient with the value of $156 \mathrm{~mm}^{-1} \mathrm{~cm}^{-1}$. 


\begin{tabular}{|c|c|c|c|c|c|c|c|c|c|}
\hline $\begin{array}{l}\text { Thermal } \\
\text { regimes }\end{array}$ & Nutrients & Pn 2012 & Pn 2013 & Gs 2012 & Gs 2013 & \begin{tabular}{|l} 
Water \\
Potential \\
2012
\end{tabular} & \begin{tabular}{|l} 
Water \\
Potential \\
2013
\end{tabular} & $\begin{array}{l}\text { Osmotic } \\
\text { Potential } \\
2012\end{array}$ & $\begin{array}{l}\text { Osmotic } \\
\text { Potential } \\
2013\end{array}$ \\
\hline \multirow{4}{*}{$\begin{array}{l}\text { Optimal } \\
\text { regimes } \\
\text { of sowing } \\
\text { dates }\end{array}$} & Control & $\begin{array}{l}27.43 \\
\mathrm{a} \pm 2.4\end{array}$ & $\begin{array}{l}27.27 \\
\mathrm{a} \pm 2.5\end{array}$ & $\begin{array}{l}0.81 \\
\mathrm{a} \pm 0.076\end{array}$ & $\begin{array}{l}0.81 \\
\mathrm{a} \pm 0.075\end{array}$ & $\begin{array}{l}0.45 \\
\mathrm{a} \pm 0.039\end{array}$ & $\begin{array}{l}0.46 \\
\mathrm{a} \pm 0.041\end{array}$ & $\begin{array}{l}0.70 \\
a \pm 0.065\end{array}$ & $\begin{array}{l}0.69 \\
\mathrm{a} \pm 0.062\end{array}$ \\
\hline & Potassium (1.5\%) & $\begin{array}{l}26.83 \\
\mathrm{a} \pm 2.1\end{array}$ & $\begin{array}{l}26.18 \\
\mathrm{a} \pm 2.2\end{array}$ & $\begin{array}{l}0.79 \\
\mathrm{a} \pm 0.073\end{array}$ & $\begin{array}{l}0.81 \\
\mathrm{a} \pm 0.077\end{array}$ & $\begin{array}{l}0.45 \\
\mathrm{a} \pm 0.035\end{array}$ & $\begin{array}{l}0.44 \\
\mathrm{a} \pm 0.038\end{array}$ & $\begin{array}{l}0.70 \\
\mathrm{a} \pm 0.062\end{array}$ & $\begin{array}{l}0.69 \\
\mathrm{a} \pm 0.065\end{array}$ \\
\hline & Zinc $(0.2 \%)$ & $\begin{array}{l}26.05 \\
\mathrm{a} \pm 2.3\end{array}$ & $\begin{array}{l}26.07 \\
a \pm 1.9\end{array}$ & $\begin{array}{l}0.80 \\
\mathrm{a} \pm 0.071\end{array}$ & $\begin{array}{l}0.80 \\
\mathrm{a} \pm 0.075\end{array}$ & $\begin{array}{l}0.42 \\
\mathrm{a} \pm 0.040\end{array}$ & $\begin{array}{l}0.42 \\
\mathrm{a} \pm 0.036\end{array}$ & $\begin{array}{l}0.70 \\
a \pm 0.069\end{array}$ & $\begin{array}{l}0.68 \\
\mathrm{a} \pm 0.059\end{array}$ \\
\hline & Boron $(0.1 \%)$ & $\begin{array}{l}26.01 \\
\mathrm{a} \pm 2.0\end{array}$ & $\begin{array}{l}26.20 \\
\mathrm{a} \pm 2.4\end{array}$ & $\begin{array}{l}0.80 \\
a \pm 0.077\end{array}$ & $\begin{array}{l}0.80 \\
\mathrm{a} \pm 0.074\end{array}$ & $\begin{array}{l}0.43 \\
\mathrm{a} \pm 0.038\end{array}$ & $\begin{array}{l}0.47 \\
\mathrm{a} \pm 0.043\end{array}$ & $\begin{array}{l}0.69 \\
a \pm 0.060\end{array}$ & $\begin{array}{l}0.68 \\
\mathrm{a} \pm 0.061\end{array}$ \\
\hline \multirow{4}{*}{$\begin{array}{l}\text { Supra- } \\
\text { optimal } \\
\text { of sowing } \\
\text { dates }\end{array}$} & Control & $\begin{array}{l}18.06 \\
c \pm 1.6\end{array}$ & $\begin{array}{l}18.92 \\
c \pm 1.3\end{array}$ & $\begin{array}{l}0.52 \\
c \pm 0.049\end{array}$ & $\begin{array}{l}0.53 \\
c \pm 0.049\end{array}$ & $\begin{array}{l}0.67 \\
\mathrm{a} \pm 0.057\end{array}$ & $\begin{array}{l}0.64 \\
\mathrm{a} \pm 0.059\end{array}$ & $\begin{array}{l}1.07 \\
\mathrm{a} \pm 0.091\end{array}$ & $\begin{array}{l}1.04 \\
\mathrm{a} \pm 0.091\end{array}$ \\
\hline & Potassium (1.5\%) & $\begin{array}{l}24.62 \\
\mathrm{a} \pm 1.9\end{array}$ & $\begin{array}{l}25.20 \\
\mathrm{a} \pm 1.8\end{array}$ & $\begin{array}{l}0.73 \\
\mathrm{a} \pm 0.068\end{array}$ & $\begin{array}{l}0.74 \\
\mathrm{a} \pm 0.069\end{array}$ & $\begin{array}{l}0.49 \\
c \pm 0.042\end{array}$ & $\begin{array}{l}0.46 \\
c \pm 0.044\end{array}$ & $\begin{array}{l}0.78 \\
c \pm 0.072\end{array}$ & $\begin{array}{l}0.75 \\
c \pm 0.070\end{array}$ \\
\hline & Zinc $(0.2 \%)$ & $\begin{array}{l}23.76 \\
\mathrm{a} \pm 1.8\end{array}$ & $\begin{array}{l}24.26 \\
\mathrm{a} \pm 2.1\end{array}$ & $\begin{array}{l}0.73 \\
a \pm 0.069\end{array}$ & $\begin{array}{l}0.73 \\
\mathrm{a} \pm 0.071\end{array}$ & $\begin{array}{l}0.50 \\
c \pm 0.046\end{array}$ & $\begin{array}{l}0.46 \\
c \pm 0.041\end{array}$ & $\begin{array}{l}0.78 \\
c \pm 0.068\end{array}$ & $\begin{array}{l}0.75 \\
c \pm 0.068\end{array}$ \\
\hline & Boron $(0.1 \%)$ & $\begin{array}{l}21.56 \\
\mathrm{ab} \pm 1.6\end{array}$ & $\begin{array}{l}22.10 \\
\mathrm{ab} \pm 1.9\end{array}$ & $\begin{array}{l}0.63 \\
b \pm 0.057\end{array}$ & $\begin{array}{l}0.63 \\
\mathrm{~b} \pm 0.058\end{array}$ & $\begin{array}{l}0.59 \\
\mathrm{~b} \pm 0.053\end{array}$ & $\begin{array}{l}0.55 \\
\mathrm{~b} \pm 0.051\end{array}$ & $\begin{array}{l}0.88 \\
\mathrm{~b} \pm 0.082\end{array}$ & $\begin{array}{l}0.86 \\
b \pm 0.081\end{array}$ \\
\hline \multirow{5}{*}{$\begin{array}{l}\text { Sub- } \\
\text { optimal } \\
\text { of sowing } \\
\text { dates }\end{array}$} & Control & $\begin{array}{l}24.58 \\
\mathrm{ab} \pm 2.2\end{array}$ & $\begin{array}{l}23.99 \\
\mathrm{ab} \pm 2.2\end{array}$ & $\begin{array}{l}0.78 \\
\mathrm{a} \pm 0.073\end{array}$ & $\begin{array}{l}0.77 \\
\mathrm{a} \pm 0.071\end{array}$ & $\begin{array}{l}0.44 \\
\mathrm{a} \pm 0.038\end{array}$ & $\begin{array}{l}0.42 \\
\mathrm{a} \pm 0.037\end{array}$ & $\begin{array}{l}0.65 \\
a \pm 0.057\end{array}$ & $\begin{array}{l}0.68 \\
\mathrm{a} \pm 0.061\end{array}$ \\
\hline & Potassium (1.5\%) & $\begin{array}{l}26.83 \\
\mathrm{a} \pm 2.5\end{array}$ & $\begin{array}{l}26.56 \\
\mathrm{a} \pm 2.5\end{array}$ & $\begin{array}{l}0.80 \\
a \pm 0.076\end{array}$ & $\begin{array}{l}0.80 \\
\mathrm{a} \pm 0.075\end{array}$ & $\begin{array}{l}0.44 \\
\mathrm{a} \pm 0.041\end{array}$ & $\begin{array}{l}0.42 \\
\mathrm{a} \pm 0.038\end{array}$ & $\begin{array}{l}0.64 \\
\mathrm{a} \pm 0.059\end{array}$ & $\begin{array}{l}0.68 \\
\mathrm{a} \pm 0.058\end{array}$ \\
\hline & Zinc $(0.2 \%)$ & $\begin{array}{l}27.80 \\
\mathrm{a} \pm 2.6\end{array}$ & $\begin{array}{l}26.41 \\
\mathrm{a} \pm 2.3\end{array}$ & $\begin{array}{l}0.79 \\
\mathrm{a} \pm 0.074\end{array}$ & $\begin{array}{l}0.81 \\
\mathrm{a} \pm 0.079\end{array}$ & $\begin{array}{l}0.41 \\
\mathrm{a} \pm 0.038\end{array}$ & $\begin{array}{l}0.39 \\
\mathrm{a} \pm 0.033\end{array}$ & $\begin{array}{l}0.64 \\
\mathrm{a} \pm 0.062\end{array}$ & $\begin{array}{l}0.68 \\
\mathrm{a} \pm 0.063\end{array}$ \\
\hline & Boron $(0.1 \%)$ & $\begin{array}{l}26.92 \\
\mathrm{a} \pm 2.2\end{array}$ & $\begin{array}{l}27.19 \\
\mathrm{a} \pm 2.6\end{array}$ & $\begin{array}{l}0.78 \\
a \pm 0.070\end{array}$ & $\begin{array}{l}0.77 \\
\mathrm{a} \pm 0.071\end{array}$ & $\begin{array}{l}0.41 \\
\mathrm{a} \pm 0.037\end{array}$ & $\begin{array}{l}0.39 \\
\mathrm{a} \pm 0.035\end{array}$ & $\begin{array}{l}0.63 \\
a \pm 0.059\end{array}$ & $\begin{array}{l}0.67 \\
\mathrm{a} \pm 0.061\end{array}$ \\
\hline & \begin{tabular}{|l|} 
LSD \\
\end{tabular} & 2.98 & 3.01 & 0.096 & 0.089 & 0.08 & 0.073 & 0.10 & 0.11 \\
\hline
\end{tabular}

Table 7. Effect of different thermal regimes and nutrients' spray on net photosynthetic rate-Pn $\left(\mu \mathrm{mol} \mathrm{m}{ }^{-2}\right.$ $\left.\left.\mathrm{sec}^{-1}\right), \mathrm{FW}\right)$, stomatal conductance $\left(\mathrm{Gs} \mathrm{m} \mathrm{mol} \mathrm{m} \mathrm{m} \mathrm{s}^{-1}\right)$, leaf water potential (-MPa) and leaf osmotic potential ( $-\mathrm{MPa}$ ) (averaged across of squaring, flowering and boll formation stages) of cotton leaves under field conditions during 2012 and 2013. Values are the means of three replications $(n=3) \pm S E$ and variants possessing the same letters are not statistically significant at $P<0.05$. Lettering is done separately for each thermal regime using the LSD of the interaction between thermal regimes and nutrients' spray.

\begin{tabular}{|c|c|c|c|c|c|}
\hline Thermal regimes & Nutrients & $\begin{array}{l}\text { Boll weight } \\
\text { (g) } 2012\end{array}$ & $\begin{array}{l}\text { Boll weight } \\
\text { (g) } 2013\end{array}$ & SCY 2012 & SCY 2013 \\
\hline \multirow{4}{*}{$\begin{array}{l}\text { April (High } \\
\text { temperature) sub } \\
\text { optimal }\end{array}$} & Control & $3.53 \mathrm{a} \pm 0.31$ & $3.64 \mathrm{~b} \pm 0.32$ & $87.60 \mathrm{~b} \pm 8.4$ & $85.60 \mathrm{~b} \pm 8.1$ \\
\hline & Potassium (1.5\%) & $4.13 \mathrm{a} \pm 0.38$ & $4.24 \mathrm{a} \pm 0.39$ & $101.34 \mathrm{a} \pm 9.9$ & $99.34 \mathrm{a} \pm 9.4$ \\
\hline & Zinc $(0.2 \%)$ & $4.05 \mathrm{a} \pm 0.40$ & $4.16 \mathrm{a} \pm 0.37$ & $98.44 \mathrm{a} \pm 9.2$ & $97.62 \mathrm{a} \pm 9.2$ \\
\hline & Boron $(0.1 \%)$ & $4.07 \mathrm{a} \pm 0.35$ & $4.18 \mathrm{a} \pm 0.41$ & $99.41 \mathrm{a} \pm 9.5$ & $97.41 \mathrm{a} \pm 9.6$ \\
\hline \multirow{4}{*}{$\begin{array}{l}\text { May (High } \\
\text { temperature) } \\
\text { supra }\end{array}$} & Control & $2.88 \mathrm{~b} \pm 0.23$ & $2.99 \mathrm{~b} \pm 0.26$ & $70.11 \mathrm{~b} \pm 6.3$ & $68.45 \mathrm{~b} \pm 6.4$ \\
\hline & Potassium (1.5\%) & $3.41 \mathrm{a} \pm 0.29$ & $3.52 \mathrm{a} \pm 0.32$ & $82.89 \mathrm{a} \pm 7.7$ & $81.22 \mathrm{a} \pm 7.8$ \\
\hline & Zinc $(0.2 \%)$ & $3.36 \mathrm{a} \pm 0.31$ & $3.46 \mathrm{a} \pm 0.29$ & $82.37 \mathrm{a} \pm 7.9$ & $81.37 \mathrm{a} \pm 7.5$ \\
\hline & Boron $(0.1 \%)$ & $3.35 \mathrm{a} \pm 0.30$ & $3.45 \mathrm{a} \pm 0.27$ & $80.92 \mathrm{a} \pm 7.5$ & $79.92 \mathrm{a} \pm 7.1$ \\
\hline \multirow{5}{*}{$\begin{array}{l}\text { June (late sown as } \\
\text { optimal) }\end{array}$} & Control & $2.61 \mathrm{a} \pm 0.20$ & $2.72 \mathrm{a} \pm 0.21$ & $49.99 \mathrm{a} \pm 4.3$ & $51.19 \mathrm{a} \pm 4.7$ \\
\hline & Potassium (1.5\%) & $2.66 \mathrm{a} \pm 0.24$ & $2.78 \mathrm{a} \pm 0.25$ & $52.99 \mathrm{a} \pm 4.7$ & $54.19 \mathrm{a} \pm 4.9$ \\
\hline & Zinc $(0.2 \%)$ & $2.67 \mathrm{a} \pm 0.22$ & $2.79 \mathrm{a} \pm 0.24$ & $47.07 \mathrm{a} \pm 4.1$ & $48.27 \mathrm{a} \pm 4.5$ \\
\hline & Boron $(0.1 \%)$ & $2.61 \mathrm{a} \pm 0.18$ & $2.73 \mathrm{a} \pm 0.26$ & $47.33 \mathrm{a} \pm 4.0$ & $48.53 \mathrm{a} \pm 4.3$ \\
\hline & LSD & 0.37 & 0.40 & 9.66 & 9.78 \\
\hline
\end{tabular}

Table 8. Effect of different thermal regimes and nutrients' spray on averaged boll weight ( $\mathrm{g}$ ) and seed cotton yield per plant (g) of cotton crop under field conditions during 2012 and 2013 . Values are the means of three replications $(n=3) \pm S E$ and variants possessing the same letters are not statistically significant at $P<0.05$. Lettering is done separately for each thermal regime using the LSD of the interaction between thermal regimes and nutrients' spray.

Chlorophyll contents. Cotton leaves $(0.5 \mathrm{~g})$ were ground in $10 \mathrm{ml}$ of $80 \%$ cold acetone and the tubes were stored in dark at $20^{\circ} \mathrm{C}$ overnight, indicating minor modifications of previously described method ${ }^{33}$. The mixture was filtered through a Whatman No 1 . A blank with $80 \%$ acetone was run; the measurements were taken at 645 and $663 \mathrm{~nm}$ through a spectrophotometer. The chlorophyll contents were calculated from the formula: 
(a)

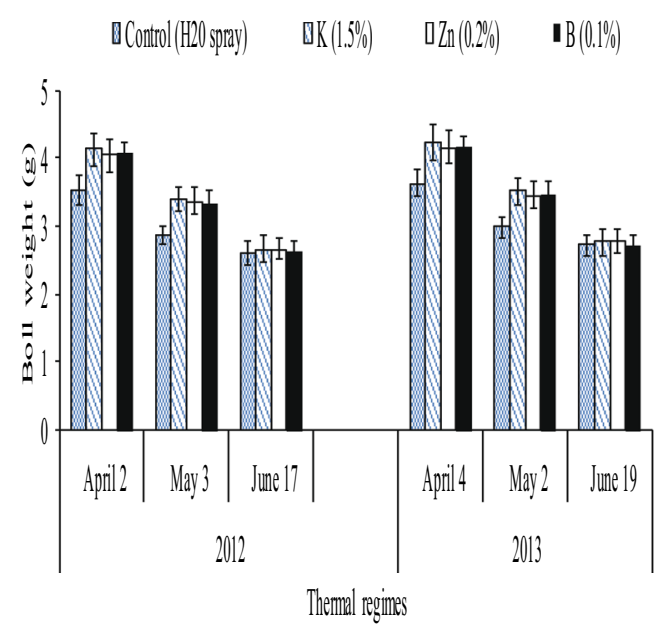

(b)

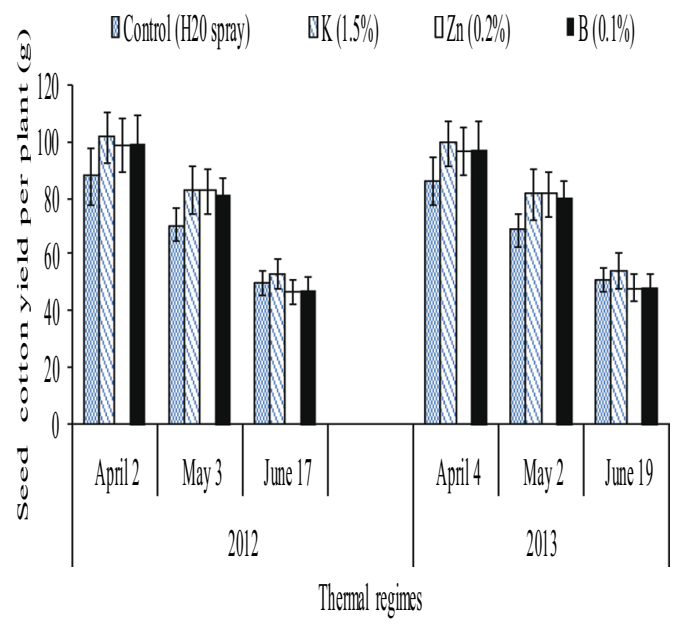

Figure 6. Effect of different thermal regimes and nutrients' spray on averaged boll weight $(\mathrm{g})$ and seed cotton yield per plant $(\mathrm{g})$ of cotton crop under field conditions during 2012 and 2013.

$$
\begin{aligned}
& \text { Chl } a\left(\frac{m g}{g} F W\right)=[12.7(O D 663)-2.69(O D \text { 645) })] * \frac{V}{1000} * W \\
& \text { Chl b }\left(\frac{m g}{g} F W\right)=\left[22.9(O D \text { 645 })-4.68(O D \text { 663) }] * \frac{V}{1000} * W\right.
\end{aligned}
$$

where $\mathrm{W}$ is the weight of leaf sample while $\mathrm{V}$ is the volume of sample used in spectrophotometer (U-2001, Hitachi, Japan).

Net photosynthetic rate and stomatal conductance. Net photosynthetic rate and stomatal conductance was determined at three reproductive stages of cotton crop through a portable infrared gas analyzer (LCiAnalyser having Broad Head, Part Number LCi-002/B with Serial Number 32455). The Pn was measured at each reproductive stage after 3 days of spray between 10:00 a.m. to 12:00 p.m. on fully expanded young leaves.

Water relations. Leaf samples (Leaf water and osmotic potential) were collected at pre-dawn $(6: 00 \mathrm{~h})$ as previously described ${ }^{34}$. Leaf water potential was determined through Scholander type pressure chamber (ARIMAD 2, Korea) following methodology as described by ${ }^{35}$ instantly after sampling. While, leaves were stored at $-20^{\circ} \mathrm{C}$ for a period of one week, then thawed, extracted sap and determined the osmotic potential with the help of osmometer (Osmomat 030).

Agronomic attributes. Ten plants were randomly selected in each experimental unit of filed study while five plants were selected from five random pots of optimal, sub and supra-optimal thermal regimes of glass house study. Averaged boll weight was noted by dividing total seed cotton yield per plant with total number of bolls. While, seed cotton yield was weighed separately for each plot/pot and converted to per hectare yield from each plot.

Statistical analysis. Analysis of variance was employed to determine significance (F-test) of heat and foliar nutrients. While, means of treatments were compared using least significant difference test $(\mathrm{p} \leq 0.05)$. Correlation among the varying response variables was computed using means of treatments calculated across the three blocks. Strength, type and significance of correlation was determined using STATISTIX 8.1 software (Analytical Software, Tallahassee, Florida, USA). Number of pairs of observations (n) to determine correlation were 36 (replications $\times$ main plots $\times$ sub plots). Figures were developed using MS excel-2016.

\section{Results}

Green house experiment. Significant interaction of heat and foliar nutrients was recorded for all the studied attributes. Supra optimal regime followed by sub optimal regime triggered increase in antioxidants, MDA and decrease in chlorophyll contents, photosynthetic rate, gaseous exchange components, water relations, boll weight and seed cotton yield over the optimal temperature regime. Foliar applied ' $0.2 \% \mathrm{Zn}$ ' depicted outstanding results 


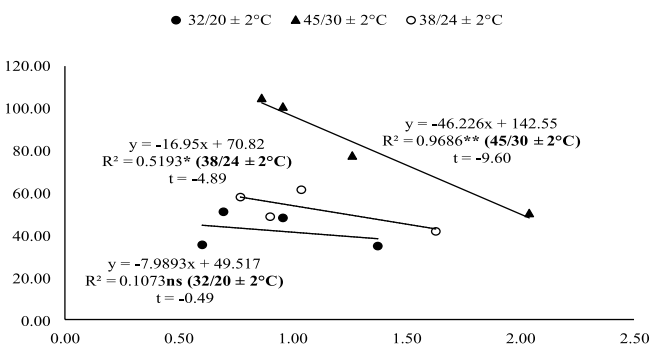

(a)

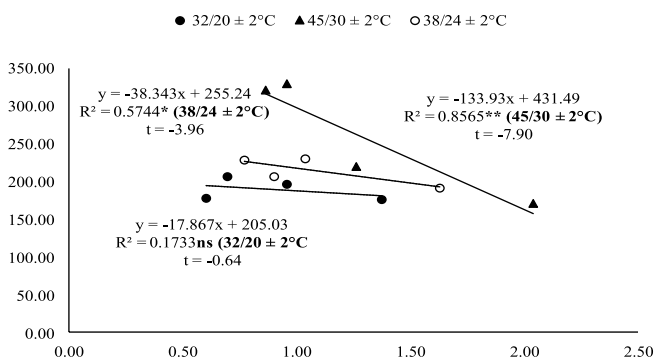

(b)

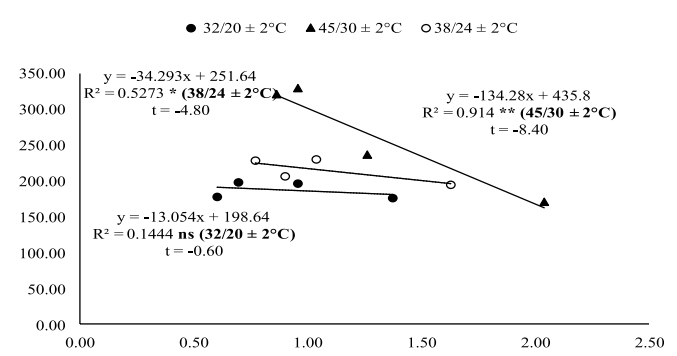

(c)

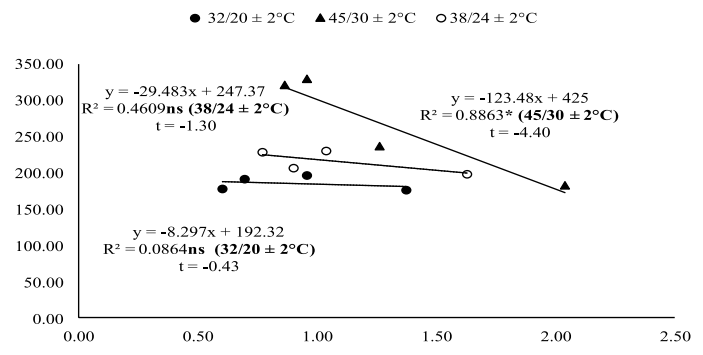

(d)

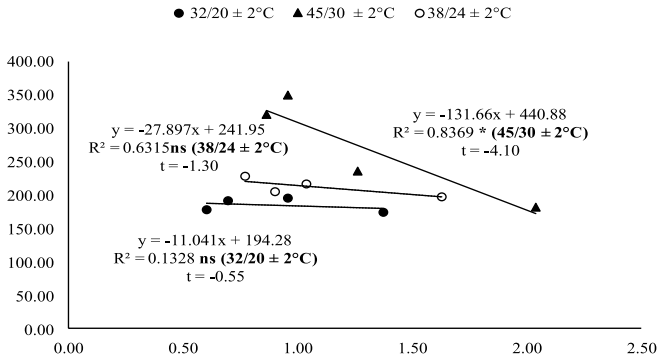

(e)

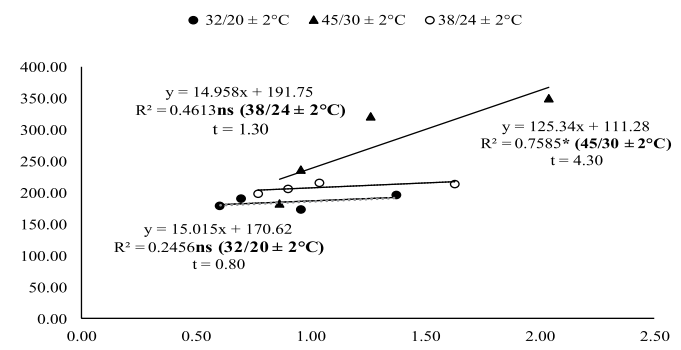

(f)

Figure 7. Association between malondialdehyde with (a) peroxidase, (b) ascorbic acid and (c) total phenolic contents, (d) stomatal conductance, (e) boll weight and (f) of stomatal conductance with water potential under glass house conditions (averaged across of three reproductive stages). * and ** indicates significance at 5 and $1 \%$ levels, respectively.

regarding the alleviation of adverse impacts of heat, followed by ' $1.5 \% \mathrm{~K}$ ' and ' $0.1 \% \mathrm{~B}$ ' for all the studied attributes (Tables 2-4, Figs 1-3).

Superoxide dismutase contents were improved by $46 \%$ and $25 \%$ while catalase contents were increased by $61 \%$ and $29 \%$ when compared the controls of supra and sub-optimal thermal regimes with the control of optimal thermal regime averaged across of three reproductive stages. Similarly, POX, AsA and TPC contents were increased under sub and supra-optimal thermal regimes. Biosynthesis of SOD was enhanced by $32 \%$ and $56 \%$ with ' $0.2 \% \mathrm{Zn}$ ' compared to water spray under sub and supra-optimal thermal regimes. Similarly, ' $0.2 \% \mathrm{Zn}$ ' instigated improvements in biosynthesis of CAT by $60 \%$ and $73 \%$ under sub and supra-optimal temperature regime than their respective water treated plants. The SOD and CAT contents were also increased under optimal thermal regime but the effect was more pronounced under sub and supra-optimal thermal regimes. Whereas, POX was up-regulated by 31,32 and 52\% under optimal, sub optimal and supra optimal temperature regimes, respectively with ' $0.2 \% \mathrm{Zn}$ ' compared to water spray. Likewise, Zn also enhanced the POX, AsA and TPC contents under optimal, sub optimal and supra optimal temperature regimes. Moreover, MDA synthesis was downregulated by $56 \%$, $58 \%$ and $57 \%$ with ' $0.2 \%$ Zn' compared to 'water treated plants' under optimal, sub optimal and supra optimal temperature regimes, respectively (Table 2, Fig. 1).

The Chlorophyll $a$ and $b$ contents were reduced by $15 \%$ and $66 \%$ under the controls (water treated plants) of sub and supra-optimal thermal regimes when compared with water treated plants of optimal thermal regime. Net photosynthetic rate was reduced by $20 \%$ and $60 \%$ when compared the water treated plants of sub and supra-optimal thermal regimes with water treated plants of optimal thermal regime (averaged across of three reproductive stages). Similarly, stomatal conductance and water potential were reduced while osmotic potential was increased under sub and supra optimal thermal regimes.

The comparative improvements in chlorophyll $a, b$ contents, Pn and Gs owing to ' $0.2 \%$ Zn' with respect to water spray were statistically higher under sub and supra optimal thermal regimes. For example, $\mathrm{Zn}$ improved Chlorophyll $a$ content by $23 \%$ and $46 \%$ under sub and supra optimal thermal regimes than water treated plants. 


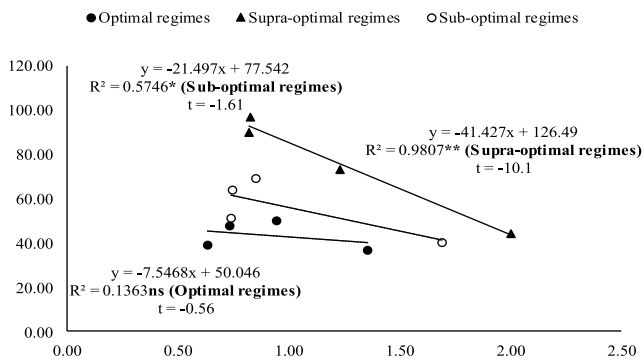

(a)

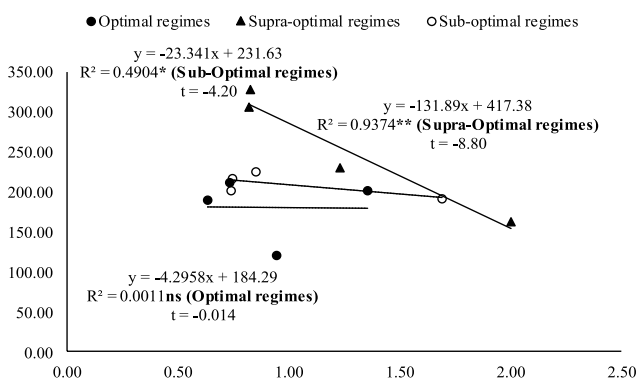

(b)

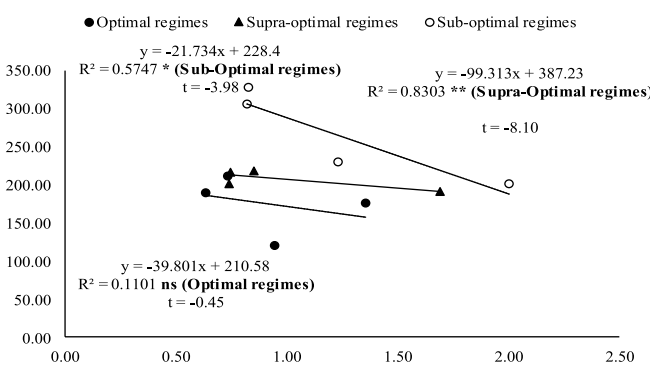

(c)

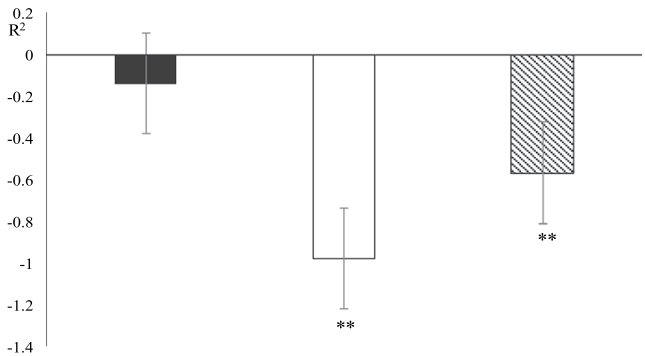

(d)

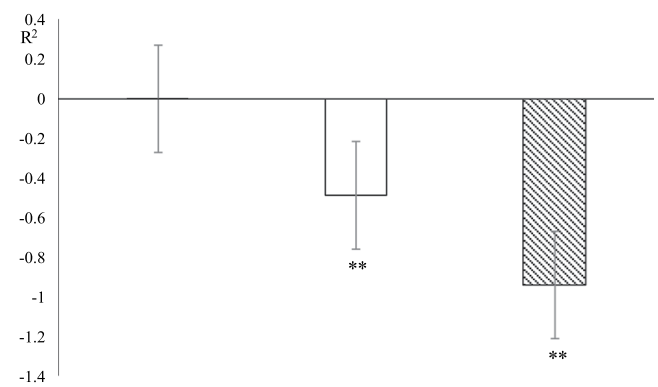

(e)

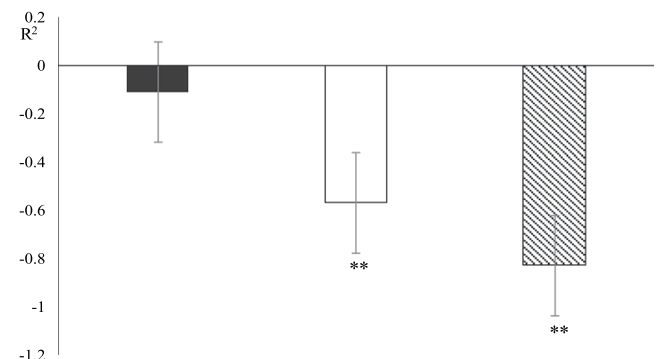

(f)

Figure 8. Association between malondialdehyde with (a) peroxidase, (b) ascorbic acid and (c) total phenolic contents under field conditions (averaged across of three reproductive stages). $*$ and $* *$ indicates significance at 5 and $1 \%$ levels, respectively.

Similarly, zinc also improved chlorophyll $b$ contents, Pn, Gs and water potential under sub and supra optimal thermal regimes. (Table 3, Fig. 2).

Although, the seed cotton yield (SCY) was reduced by $66 \%$ and $23 \%$ in the controls of supra and sub-optimal thermal regimes than the control of optimal thermal regime. The similar reduction was found for averaged boll weight. The foliar spray of three nutrients (K, Zn and B) improved SCY by $21 \%, 16 \%$ and $7 \%$ in the high temperature regime than water treated plants. Likewise, the nutrients improved the averaged boll weight under high temperature regime (Table 4, Fig. 3).

Field experiment. Supra optimal temperature regimes were relatively more detrimental, and it was followed by sub optimal temperature regimes (Table 1). While, exogenously applied nutrients depicted significant improvements compared to water spray (control). However, relatively more promising results were obtained with ' $0.2 \% \mathrm{Zn}$, followed by ' $1.5 \% \mathrm{~K}$, ' $0.1 \% \mathrm{~B}$ ' and water spray. The recorded improvements by the application of exogenous nutrients differ significantly under varying temperature regimes. (Tables 5-8, Figs 4-6).

In the controls of supra and sub optimal thermal regimes of April and May sown crops, the SOD and CAT contents were increased by $37 \%, 36 \%$ and $22 \%, 11 \%$ (averaged across both years of study and of three developmental stages) than the water treated plants of optimal thermal regime. Significantly higher activities of SOD, CAT, POX, AsA and TPC with ' $0.2 \%$ Zn' compared to foliar spray of other nutrients were quantified under all temperature regimes for most of cases over the years. However, ' $0.2 \% \mathrm{Zn}$ ' mediated improvements in biosynthesis of SOD compared to water spray were $32 \%$ under sub optimal and $49 \%$ under supra optimal temperature regimes averaged across both years of study. Likewise, CAT, POX, AsA and TPC contents were improved by foliar spray of zinc under sub and supra optimal thermal regimes over water treated plant (Tables 5 and 6, Fig. 4). Statistically significant decrease in MDA contents were observed with ' $0.2 \% \mathrm{Zn}$ ' and ' $1.5 \% \mathrm{~K}$ ' compared to other exogenous treatments under varying temperature regimes. However, remarkable change in biosynthesis of MDA was recorded with ' $0.2 \% \mathrm{Zn}$ ' compared to other sprays. Foliar spray of ' $0.2 \% \mathrm{Zn}$ ' instigated downregulation in 

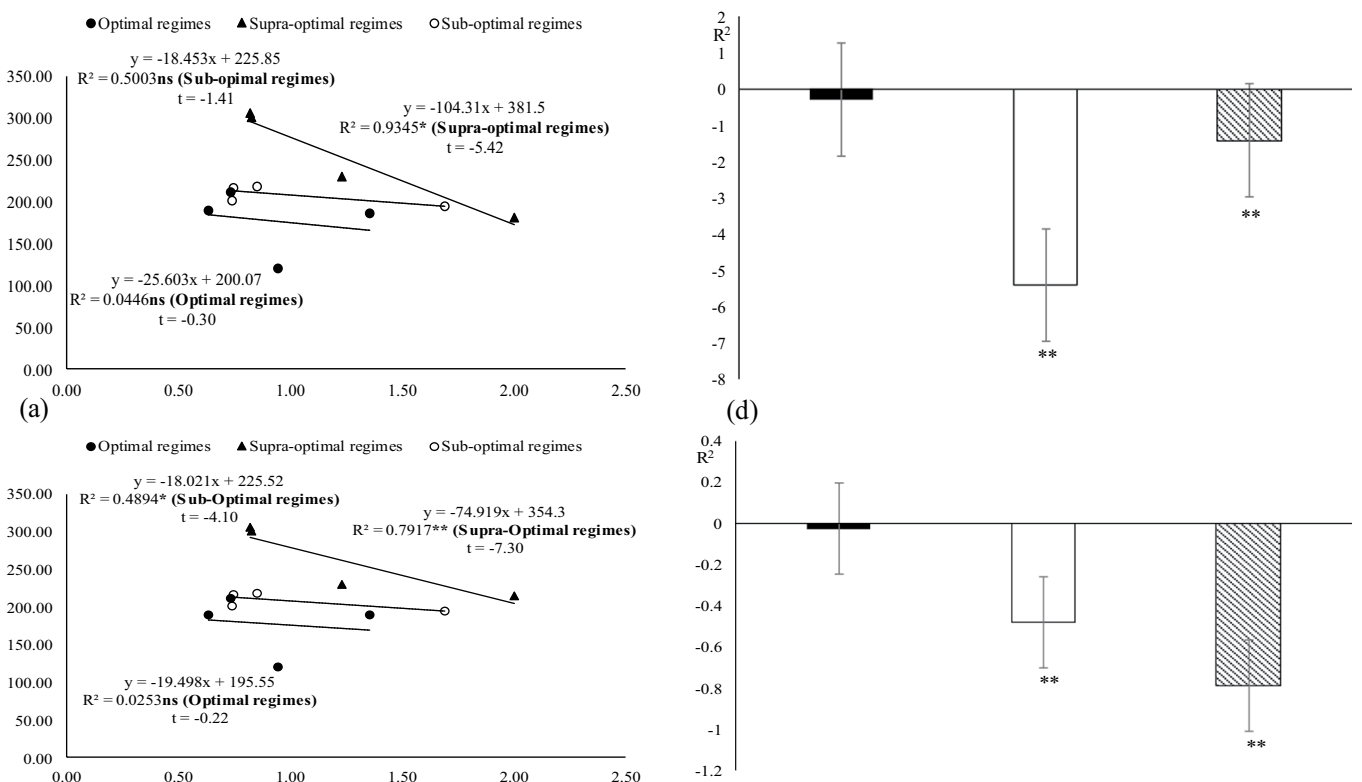

(d)

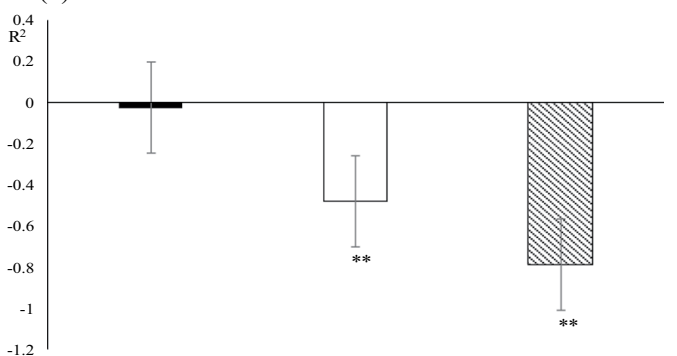

(b)

(e)
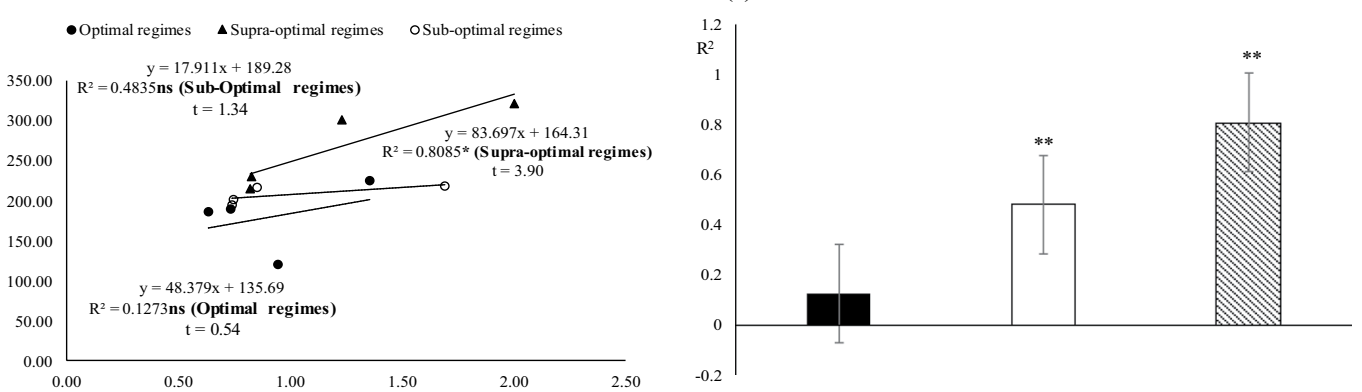

(c)

(f)

Figure 9. Association between malondialdehyde with (a) stomatal conductance, (b) boll weight and (c) of stomatal conductance with water potential under field conditions (averaged across of three reproductive stages). $*$ and $* *$ indicates significance at 5 and $1 \%$ levels, respectively.

\begin{tabular}{|l|l|l|l|l|l|}
\hline $\begin{array}{l}\text { Correlation } \\
\text { components }\end{array}$ & CAT & Chlor a & Chlor b & Pn & SCY \\
\hline Chlor a & $0.47^{*}$ & & & & \\
\hline Chlor b & $0.48^{*}$ & $0.96^{* *}$ & & & \\
\hline Pn & $0.51 *$ & $0.98^{* *}$ & $0.98^{* *}$ & & \\
\hline SCY & $0.89 * *$ & $0.71 * *$ & $0.69 * *$ & $0.68^{* *}$ & \\
\hline SOD & $0.76^{* *}$ & $0.85^{* *}$ & $0.82^{* *}$ & $0.85 * *$ & $0.67 * *$ \\
\hline
\end{tabular}

Table 9. Correlation between Chlor.a/b, Pn, CAT, SOD and SCY under glass house conditions. Chlor a (Chlorophyll a), Chlor b (Chlorophyll b), CAT (Catalase), SOD (Superoxide dismutase), Pn (Net photosynthetic rate) and SCY (Seed cotton yield). *Correlation is significant at 0.01 levels. **Correlation is significant at 0.05 level. $\mathrm{n}$ (number of pairs of observations) $=48$.

MDA contents compared to water spray were 52\% under sub optimal and 59\% under supra optimal temperature regimes averaged across both years of study (Table 6, Fig. 4).

Chlorophyll $a, b$ contents were reduced by $66 \%, 51 \%$ and $16 \%, 13 \%$, respectively in the controls of supra and sub-optimal thermal regimes than the controls of optimal thermal regime averaged across of both years and of three development stages. Statistically alike and significantly more chlorophyll $a$ and $b$ biosynthesis was quantified with ' $0.2 \% \mathrm{Zn}$ ' and ' $1.5 \% \mathrm{~K}$ ' compared to other foliar sprays under supra optimal temperature regime (Table 6, Fig. 5).

The Pn was reduced by $47 \%$ and $13 \%$ under the controls of supra and sub-optimal thermal regimes than the control of optimal thermal regime averaged across during both years of study and of three reproductive stages. Significantly more net photosynthetic rate, stomatal conductance, water potential and less osmotic potential were 


\begin{tabular}{|c|c|c|c|c|c|c|}
\hline Parameters & Years & CAT & Chl a & Chl b & Pn & SCY \\
\hline \multirow{2}{*}{ Chl a } & 2012 & $0.74^{* *}$ & & & & \\
\hline & 2013 & $0.89 * *$ & & & & \\
\hline \multirow{2}{*}{$\mathrm{Chl} \mathrm{b}$} & 2012 & $0.67 * *$ & $0.94 * *$ & & & \\
\hline & 2013 & $0.77 * *$ & $0.75 * *$ & & & \\
\hline \multirow{2}{*}{ Pn } & 2012 & $0.85^{* *}$ & $0.90 * *$ & $0.63 * *$ & & \\
\hline & 2013 & $0.72 * *$ & $0.86 * *$ & $0.68^{* *}$ & & \\
\hline \multirow{2}{*}{ SCY } & 2012 & $0.69^{* *}$ & $0.70 * *$ & $0.66^{* * *}$ & 0.80 ** & \\
\hline & 2013 & $0.67 * *$ & $0.74^{* *}$ & $0.59^{*}$ & $0.89 * *$ & \\
\hline \multirow{2}{*}{ SOD } & 2012 & $0.87^{* *}$ & $0.65 * *$ & $0.71^{* *}$ & $0.52 *$ & $0.63 * *$ \\
\hline & 2013 & $0.89 * *$ & $0.65 * *$ & $0.84^{* * *}$ & $0.79 * 8$ & $0.69 * *$ \\
\hline
\end{tabular}

Table 10. Correlation between Chlor.a/b, Pn, CAT, SOD and SCY under field conditions during 2012 and 2013. Chlor a (Chlorophyll a), Chlor b (Chlorophyll b), CAT (Catalase), SOD (Superoxide dismutase), Pn (Net photosynthetic rate) and SCY (Seed cotton yield). *Correlation is significant at 0.01 levels. **Correlation is significant at 0.05 levels. $\mathrm{n}$ (number of pairs of observations) $=36$.

recorded with foliar ' $0.2 \% \mathrm{Zn}$ ' and ' $1.5 \% \mathrm{~K}$ ' under supra optimal temperature regime over two years of experimentation (Table 7, Fig. 5).

The three nutrients (K, Zn and B averaged across) increased SCY under April and May thermal regimes by $15 \%$ and $17 \%$ than water spray averaged across during both years of study (Table 8, Fig. 6).

Regression and correlation of studied components under both glasshouse and field conditions during 2012 and 2013. The regression analysis under glasshouse conditions indicated that the malondialdehyde has strong negative relation with POX, AsA, TPC and with stomatal conductance. While water relations have positive relation with stomatal conductance (Fig. 7). The regression analysis under field conditions indicated that the malondialdehyde has strong negative relation with POX, AsA, TPC and with stomatal conductance while water relations have strong positive relation with stomatal conductance (Figs 8 and 9).

In glasshouse, Chlorophyll $a$ and $b$ have significant positive relationship with each other $(P<0.05$ and $P<0.01)$ and with CAT, SOD, Pn and SCY $(P<0.05$ and $P<0.01)$. Similarly, Pn and SCY have significantly positive relation with CAT, SOD and with chlorophyll $a$ and $b$ contents (Table 9). While in filed conditions, Chlorophyll $a$ and $b$ have significant positive relationship with each other $(P<0.05$ and $P<0.01)$ and with CAT, SOD, Pn and SCY $(P<0.05$ and $P<0.01)$. Similarly, Pn and SCY have significantly positive relation with CAT, SOD and with chlorophyll a, b contents (Table 10).

Regression and correlation of studied components under glass house conditions.

\section{Discussion}

Medium high to high temperature regimes influenced cotton crop physiology and the yield ${ }^{14}$. Reactive oxygen species (ROS) affected the membranes of each organelle for example the integrity of chloroplast/photosynthetic machinery ${ }^{36,37}$. A balance is required for ROS and antioxidants for the normal functions of plant defensive system. The oxidative stress causes the blockage of nutrients channels ${ }^{5,38}$. The high temperature regimes $\left(45 / 30^{\circ} \mathrm{C}\right)$ of glass house and (April and May) of field study upregulated the superoxide dismutase, catalase, peroxidase, ascorbic acid and total phenolic contents. However, cotton plants were unable to protect the cells from MDA contents due to oxidative stress ${ }^{6}$ indicating that the optimum temperature for biochemical and metabolic functions ranges between 25 to $31^{\circ} \mathrm{C}^{39}$. In this study, the high temperature stress reduced chlorophyll contents, net photosynthetic rate and stomatal conductance ${ }^{40,41}$ indicating that photosynthetic apparatus is most sensitive to heat stress ${ }^{42}$. It may be the outcome of breakdown of photosynthetic pigments, associated proteins ${ }^{43}$, reduction in membrane integrity ${ }^{44}$, inefficiency of PS-II due to disruption of thylakoid structure ${ }^{45}$ and of linked enzymes ${ }^{46}$. The reduction in stomatal conductance under high temperature stress ${ }^{47}$ might be due to the stress signals from roots and consequent production of ethylene ${ }^{48}$ (Tables 3 and 7, Figs 2 and 5). In this study, leaf water potential was decreased while osmotic potential was increased under high temperature stress, as reported by ${ }^{11}$. It might be due to roots inability to up take water and nutrients under high temperature ${ }^{49,50}$. High temperature regimes of glass house and high temperature regimes (April and May thermal regimes) of field study showed reduction in averaged boll weight and seed cotton yield per plant as reported by ${ }^{14}$. This may be due to the production of stress hormones ${ }^{51,52}$ which may decrease the production and translocation of photo assimilates for developing bolls ${ }^{53,54}$.

The foliar spray of $\mathrm{K}, \mathrm{Zn}$ and Boron activated the plant defensive system and reduced the MDA contents from medium to high temperature regimes (Tables 2 and 6; Figs 1 and 5) as reported by ${ }^{21,55,56}$. This may be due to the reduction in oxidative stress and the stress hormones ${ }^{24,57}$.

In this study, foliar application of $\mathrm{K}, \mathrm{Zn}$ and B improved the light harvesting pigments (chlorophyll $a$ and $b$ contents $)^{58-60}$ (Tables 3 and 6, Figs 2 and 5). This may be due to the alleviation of adverse impacts of oxidative stress that may boost the chlorophyll fluorescence and the energy output of light reactions ${ }^{61,62}$.

In this study, both potassium and zinc enhanced net photosynthetic rate and stomatal conductance ${ }^{63,64}$ might be due to the role of these nutrients in $\mathrm{CO}_{2}$ assimilation and in photosynthetic process ${ }^{65-67}$.

Foliar spray of potassium, zinc and boron increased water potential and reduced solute potential ${ }^{68-70}$ as found in this study which might be due to less relative cell injury of membranes ${ }^{21,71,72}$. 
In this study, foliar spray of potassium, and zinc upsurged the boll weight and seed cotton yield even in $\mathrm{K}$ and $\mathrm{Zn}$ enriched soil ${ }^{73,74}$. This may be the outcome of the higher production of carbohydrates ${ }^{75}$. Under glass house and field studies, the chlorophyll (a and b) contents, Pn, stomatal conductance and antioxidants showed positive correlation with each other and with SCY while MDA showed strong negative relation with these parameters as reported $b^{6}$.

\section{Conclusion}

High temperature stress at three reproductive stages of cotton crop caused yield reduction which was due to lower boll weight that is associated to less chlorophyll contents and impaired photosynthesis. Exogenous application of macro and micro nutrients (K-1.5\%, $\mathrm{Zn}-0.2 \%$ and $\mathrm{B}-0.1 \%)$ ameliorated the high temperature impact on cotton crop. These nutrients especially $\mathrm{K}, \mathrm{Zn}$ and followed by B up-regulated the antioxidant enzymes (SOD, POX, CAT, AsA, phenolics and MDA), improved chlorophyll contents, net photosynthetic rate, water relations and seed cotton yield.

\section{References}

1. Dinar, A., Hassan, R., Mendelsohn, R. \& Benhin, J. Climate change and agriculture in Africa: impact assessment and adaptation strategies (2012).

2. Pachauri, R. K. et al. Climate change 2014: synthesis report. Contribution of Working Groups I, II and III to the fifth assessment report of the Intergovernmental Panel on Climate Change (2014).

3. Gür, A., Demirel, U., Özden, M., Kahraman, A. \& Çopur, O. Diurnal gradual heat stress affects antioxidant enzymes, proline accumulation and some physiological components in cotton (Gossypium hirsutum L.). African Journal of Biotechnology 9, $1008-1015$ (2010).

4. Sarwar, M. et al. Hydrogen peroxide reduces heat-induced yield losses in cotton (Gossypium hirsutum L.) by protecting cellular membrane damage. Journal of Agronomy and Crop Science (2017).

5. Hasanuzzaman, M., Nahar, K., Alam, M., Roychowdhury, R. \& Fujita, M. Physiological, biochemical, and molecular mechanisms of heat stress tolerance in plants. International journal of molecular sciences 14, 9643-9684 (2013).

6. Sarwar, M. et al. Exogenously applied growth regulators protect the cotton crop from heat-induced injury by modulating plant defense mechanism. Scientific reports 8, 17086 (2018).

7. Shahid, M., Saleem, M. F., Anjum, S. A., Shahid, M. \& Afzal, I. Effect of terminal heat stress on proline, secondary metabolites and yield components of wheat (Triticum aestivum L.) genotypes. The Philippine Agricultural Scientist 100, 278-286 (2017).

8. Pettigrew, W. Cultivar variation in cotton photosynthetic performance under different temperature regimes. Photosynthetica, 1-6 (2016).

9. Dabbert, T. \& Gore, M. A. Challenges and perspectives on improving heat and drought stress resilience in cotton. Journal of Cotton Science 18, 393-409 (2014).

10. Conaty, W., Burke, J., Mahan, J., Neilsen, J. \& Sutton, B. Determining the optimum plant temperature of cotton physiology and yield to improve plant-based irrigation scheduling. Crop Science 52, 1828-1836 (2012).

11. Wahid, A. \& Close, T. Expression of dehydrins under heat stress and their relationship with water relations of sugarcane leaves. Biologia Plantarum 51, 104-109 (2007).

12. Fahad, S. et al. Exogenously applied plant growth regulators enhance the morpho-physiological growth and yield of rice under high temperature. Frontiers in Plant Science 7 (2016).

13. Lokhande, S. \& Reddy, K. R. Quantifying temperature effects on cotton reproductive efficiency and fiber quality. Agronomy Journal 106, 1275-1282 (2014).

14. Singh, R. P., Prasad, P. V., Sunita, K., Giri, S. \& Reddy, K. R. Influence of high temperature and breeding for heat tolerance in cotton: a review. Advances in Agronomy 93, 313-385 (2007).

15. Awasthi, R. et al. Individual and combined effects of transient drought and heat stress on carbon assimilation and seed filling in chickpea. Functional Plant Biology 41, 1148-1167 (2014).

16. Waraich, E., Ahmad, R., Halim, A. \& Aziz, T. Alleviation of temperature stress by nutrient management in crop plants: a review. Journal of soil science and plant nutrition 12, 221-244 (2012).

17. Ahmad, P. \& Prasad, M. N. V. Abiotic stress responses in plants: metabolism, productivity and sustainability. (Springer Science \& Business Media, 2011).

18. Adiloglu, A. \& Adiloglu, S. The effect of boron (B) application on the growth and nutrient contents of maize in zinc (Zn) deficient soils. Research Journal of Agriculture and Biological Sciences 2, 1-4 (2006).

19. Dordas, C. Foliar boron application affects lint and seed yield and improves seed quality of cotton grown on calcareous soils. Nutrient Cycling in Agroecosystems 76, 19-28 (2006).

20. Oosterhuis, D. M. Physiological aspects of potassium deficiency in cotton. Special Reports-University Of Arkansas Agricultural Experiment Station 183, 61-73 (1997).

21. Corrales, I., Poschenrieder, C. \& Barceló, J. Boron-induced amelioration of aluminium toxicity in a monocot and a dicot species. Journal of plant physiology 165, 504-513 (2008).

22. Upadhyaya, H., Dutta, B. K. \& Panda, S. K. Zinc modulates drought-induced biochemical damages in tea [Camellia sinensis (L) O Kuntze]. Journal of agricultural and food chemistry 61, 6660-6670 (2013).

23. Zahoor, R. et al. Potassium improves photosynthetic tolerance to and recovery from episodic drought stress in functional leaves of cotton (Gossypium hirsutum L.). Plant physiology and biochemistry 119, 21-32 (2017).

24. Marschner, H. Marschner's mineral nutrition of higher plants. Academic Press Vol. 89 (2012).

25. Chen, W., Yang, X., He, Z., Feng, Y. \& Hu, F. Differential changes in photosynthetic capacity, 77 K chlorophyll fluorescence and chloroplast ultrastructure between $\mathrm{Zn}$-efficient and Zn-inefficient rice genotypes (Oryza sativa) under low zinc stress. Physiologia plantarum 132, 89-101 (2008).

26. Stavrianakou, S., Liakopoulos, G. \& Karabourniotis, G. Boron deficiency effects on growth, photosynthesis and relative concentrations of phenolics of Dittrichia viscosa (Asteraceae). Environmental and experimental botany 56, 293-300 (2006).

27. Terras, F. R. et al. A new family of basic cysteine-rich plant antifungal proteins from Brassicaceae species. FEBS letters 316, 233-240 (1993).

28. Giannopolitis, C. N. \& Ries, S. K. Superoxide dismutases I. Occurrence in higher plants. Plant physiology 59, 309-314 (1977).

29. Liu, D., Zou, J., Meng, Q., Zou, J. \& Jiang, W. Uptake and accumulation and oxidative stress in garlic (Allium sativum L.) under lead phytotoxicity. Ecotoxicology 18, 134-143 (2009).

30. Al-Yousef, S. A. Antifungal Activity of Volatiles from Lemongrass (Cymbopogon citratus) and Peppermint (Mentha piperita) Oils Against Some Respiratory Pathogenic Species of Aspergillus. Int. J. Curr. Microbiol. App. Sci 2, 261-272 (2013).

31. Ainsworth, E. A. \& Gillespie, K. M. Estimation of total phenolic content and other oxidation substrates in plant tissues using Folin-Ciocalteu reagent. Nature protocols 2, 875-877 (2007). 
32. Cakmak, I. \& Horst, W. J. Effect of aluminium on lipid peroxidation, superoxide dismutase, catalase, and peroxidase activities in root tips of soybean (Glycine max). Physiologia Plantarum 83, 463-468 (1991).

33. Arnon, D. I. Copper enzymes in isolated chloroplasts. Polyphenoloxidase in Beta vulgaris. Plant physiology 24, 1 (1949).

34. Silveira, J. A. G., Araújo, S. A. M., Lima, J. P. M. S. \& Viégas, R. A. Roots and leaves display contrasting osmotic adjustment mechanisms in response to NaCl-salinity in Atriplex nummularia. Environmental and Experimental Botany 66, 1-8 (2009).

35. Scholander, P. F., Bradstreet, E. D., Hemmingsen, E. \& Hammel, H. Sap pressure in vascular plants negative hydrostatic pressure can be measured in plants. Science 148, 339-346 (1965).

36. Mueller, M. J. \& Berger, S. Reactive electrophilic oxylipins: pattern recognition and signalling. Phytochemistry 70, 1511-1521 (2009).

37. Waszczak, C., Carmody, M. \& Kangasjärvi, J. Reactive oxygen species in plant signaling. Annual review of plant biology 69, 209-236 (2018).

38. Krieger-Liszkay, A. Singlet oxygen production in photosynthesis. Journal of experimental botany 56, 337-346 (2005).

39. Burke, J. J. \& Wanjura, D. F. In Physiology of cotton 123-128 (Springer, 2010).

40. Allakhverdiev, S. I. et al. Heat stress: an overview of molecular responses in photosynthesis. Photosynthesis research 98, 541 (2008).

41. Hemantaranjan, A. et al. Heat stress responses and thermotolerance. Adv. Plants Agric. Res 1(10), 15406 (2014).

42. Loka, D. A. \& Oosterhuis, D. M. Effect of high night temperatures during anthesis on cotton ('Gossypium hirsutum'l.) pistil and leaf physiology and biochemistry. Australian Journal of Crop Science 10, 741 (2016).

43. Kreslavski, V., Tatarinzev, N., Shabnova, N., Semenova, G. \& Kosobryukhov, A. Characterization of the nature of photosynthetic recovery of wheat seedlings from short-term dark heat exposures and analysis of the mode of acclimation to different light intensities. Journal of plant physiology 165, 1592-1600 (2008).

44. Howarth, C. Genetic improvements of tolerance to high temperature. Abiotic stresses: plant resistance through breeding and molecular approaches. Howarth Press Inc., New York (2005).

45. Zhang, X., Hegerl, G., Zwiers, F. W. \& Kenyon, J. Avoiding inhomogeneity in percentile-based indices of temperature extremes. Journal of Climate 18, 1641-1651 (2005).

46. Mathur, S. \& Jajoo, A. In Physiological mechanisms and adaptation strategies in plants under changing environment 163-191 (Springer, 2014).

47. Carmo-Silva, A. E. et al. Decreased $\mathrm{CO}_{2}$ availability and inactivation of Rubisco limit photosynthesis in cotton plants under heat and drought stress in the field. Environmental and Experimental Botany 83, 1-11 (2012).

48. Jiroutova, P., Oklestkova, J. \& Strnad, M. Crosstalk between brassinosteroids and ethylene during plant growth and under abiotic stress conditions. International journal of molecular sciences 19,3283 (2018).

49. Ghorbanpour, A., Salimi, A., Ghanbary, M. A. T., Pirdashti, H. \& Dehestani, A. The effect of Trichoderma harzianum in mitigating low temperature stress in tomato (Solanum lycopersicum L.) plants. Scientia Horticulturae 230, 134-141 (2018).

50. Pei, Z.-M. et al. Calcium channels activated by hydrogen peroxide mediate abscisic acid signalling in guard cells. Nature 406, 731-734 (2000).

51. Larkindale, J. \& Huang, B. Thermotolerance and antioxidant systems in Agrostis stolonifera: involvement of salicylic acid, abscisic acid, calcium, hydrogen peroxide, and ethylene. Journal of plant physiology 161, 405-413 (2004).

52. Ullah, A., Sun, H., Yang, X. \& Zhang, X. Drought coping strategies in cotton: increased crop per drop. Plant biotechnology journal 15, 271-284 (2017)

53. Sekmen, A. H., Ozgur, R., Uzilday, B. \& Turkan, I. Reactive oxygen species scavenging capacities of cotton (Gossypium hirsutum) cultivars under combined drought and heat induced oxidative stress. Environmental and Experimental Botany 99, 141-149 (2014).

54. Snider, J. L., Oosterhuis, D. M., Loka, D. A. \& Kawakami, E. M. High temperature limits in vivo pollen tube growth rates by altering diurnal carbohydrate balance in field-grown Gossypium hirsutum pistils. Journal of plant physiology 168, 1168-1175 (2011).

55. Han, S. et al. Boron deficiency decreases growth and photosynthesis, and increases starch and hexoses in leaves of citrus seedlings. Journal of plant physiology 165, 1331-1341 (2008).

56. Lester, G. E., Jifon, J. L. \& Makus, D. J. Impact of potassium nutrition on postharvest fruit quality: Melon (Cucumis melo L) case study. Plant and soil 335, 117-131 (2010).

57. Disante, K. B., Fuentes, D. \& Cortina, J. Response to drought of Zn-stressed Quercus suber L. seedlings. Environmental and experimental botany 70, 96-103 (2011).

58. Hu, W., Coomer, T. D., Loka, D. A., Oosterhuis, D. M. \& Zhou, Z. Potassium deficiency affects the carbon-nitrogen balance in cotton leaves. Plant physiology and biochemistry 115, 408-417 (2017).

59. Ibrahim, E. A. \& Ramadan, W. A. Effect of zinc foliar spray alone and combined with humic acid or/and chitosan on growth, nutrient elements content and yield of dry bean (Phaseolus vulgaris L.) plants sown at different dates. Scientia Horticulturae 184, 101-105 (2015)

60. Seth, K. \& Aery, N. C. Effect of boron on the contents of chlorophyll, carotenoid, phenol and soluble leaf protein in mung bean, Vigna radiata (L.) Wilczek. Proceedings of the National Academy of Sciences, India Section B: Biological Sciences 84, 713-719 (2014).

61. Eisvand, H., Kamaei, H. \& Nazarian, F. Chlorophyll fluorescence, yield and yield components of bread wheat affected by phosphate bio-fertilizer, zinc and boron under late-season heat stress. Photosynthetica 56, 1287-1296 (2018).

62. Singh, S. K. \& Reddy, V. R. Potassium starvation limits soybean growth more than the photosynthetic processes across $\mathrm{CO}_{2}$ levels. Frontiers in plant science 8, 991 (2017).

63. Hu, W. et al. Potassium (K) supply affects K accumulation and photosynthetic physiology in two cotton (Gossypium hirsutum L.) cultivars with different $\mathrm{K}$ sensitivities. Field Crops Research 196, 51-63 (2016).

64. Wang, H. \& Jin, J. Photosynthetic rate, chlorophyll fluorescence parameters, and lipid peroxidation of maize leaves as affected by zinc deficiency. Photosynthetica 43, 591-596 (2005).

65. Tsonev, T. \& Cebola Lidon, F. J. Zinc in plants-An overview. Emirates Journal of Food \& Agriculture (EJFA) 24 (2012).

66. Hu, W. et al. Potassium application affects carbohydrate metabolism in the leaf subtending the cotton (Gossypium hirsutum L.) boll and its relationship with boll biomass. Field Crops Research 179, 120-131 (2015).

67. Tsialtas, I. T., Shabala, S., Baxevanos, D. \& Matsi, T. Effect of potassium fertilization on leaf physiology, fiber yield and quality in cotton (Gossypium hirsutum L.) under irrigated Mediterranean conditions. Field crops research 193, 94-103 (2016).

68. Karim, M. R. et al. Alleviation of drought stress in winter wheat by late foliar application of zinc, boron, and manganese. Journal of Plant Nutrition and Soil Science 175, 142-151 (2012).

69. Pervez, H., Ashraf, M. \& Makhdum, M. Influence of potassium nutrition on gas exchange characteristics and water relations in cotton (Gossypium hirsutum L.). Photosynthetica 42, 251-255 (2004).

70. Sadoogh, F., Shariatmadari, H., Khoshgoftarmanesh, A. \& Mosaddeghi, M. Adjusted nutrition of tomato with potassium and zinc in drought stress conditions induced by polyethylene glycol 6000 in hydroponic culture. Journal of Science and Technology of Greenhouse Culture 5 (2014).

71. Ahanger, M. A., Agarwal, R., Tomar, N. S. \& Shrivastava, M. Potassium induces positive changes in nitrogen metabolism and antioxidant system of oat (Avena sativa L cultivar Kent). Journal of plant interactions 10, 211-223 (2015).

72. Rehman, H.-U., Aziz, T., Farooq, M., Wakeel, A. \& Rengel, Z. Zinc nutrition in rice production systems: a review. Plant and soil 361, 203-226 (2012)

73. Oosterhuis, D. M., Loka, D. A. \& Raper, T. B. Potassium and stress alleviation: Physiological functions and management of cotton. Journal of Plant Nutrition and Soil Science 176, 331-343 (2013). 
74. Soomro, A. et al. Effect of boron and zinc micronutrients on seed cotton yield and its components. Pak. J. Biol. Sci 2, 1507-1509 (2000).

75. Rashidi, M. \& Seilsepour, M. Response of yield, yield components and fiber properties of cotton to different application rates of nitrogen and boron. Journal of Environmental Science and Engineering 5, 1147-1154 (2011).

\section{Acknowledgements}

The authors are highly thankful to the University of Agriculture Faisalabad, Pakistan; the Analytical Lab, Department of Agronomy, University of Agriculture Faisalabad, Pakistan, for providing technical support and glasshouse/laboratory facilities. The authors would like to extend their sincere appreciation to the Deanship of Scientific Research at King Saud University for its funding Research group No. (RGP-199).

\section{Author Contributions}

Muhammad Sarwar, Muhammad Farrukh Saleem conducted the experiment and wrote the paper; Muhammad Rizwan, Shafaqat Ali supervised the study; Najeeb Ullah, Muhammad Rizwan Shahid, Saud A. Alamri; organized and analyzed the data Mohammed Nasser Alyemeni, Parvaiz Ahmad, provided the technical support and chemicals required for the experiment.

\section{Additional Information}

Competing Interests: The authors declare no competing interests.

Publisher's note: Springer Nature remains neutral with regard to jurisdictional claims in published maps and institutional affiliations.

(c) (i) Open Access This article is licensed under a Creative Commons Attribution 4.0 International License, which permits use, sharing, adaptation, distribution and reproduction in any medium or format, as long as you give appropriate credit to the original author(s) and the source, provide a link to the Creative Commons license, and indicate if changes were made. The images or other third party material in this article are included in the article's Creative Commons license, unless indicated otherwise in a credit line to the material. If material is not included in the article's Creative Commons license and your intended use is not permitted by statutory regulation or exceeds the permitted use, you will need to obtain permission directly from the copyright holder. To view a copy of this license, visit http://creativecommons.org/licenses/by/4.0/.

(c) The Author(s) 2019 